\title{
Sedentarismo, actividad física y salud: una revision narrativa Sedentary lifestyle, physical activity and health: a narrative review
}

\author{
Julián Camilo Garzón Mosquera y Luis Fernando Aragón Vargas
}

Universidad de Costa Rica (Costa Rica)

\begin{abstract}
Resumen. El propósito de esta revisión narrativa es ofrecer una base científicamente sólida, en español, para respaldar la promoción de la actividad física como componente indispensable de una vida saludable. La revisión está fundamentada en dos volúmenes especiales publicados recientemente en las revistas Lancet y Medicine and Science in Sports and Exercise, así como en una colección de manuscritos originales de otras fuentes. Se discuten los conceptos de sedentarismo, tiempo sentado y tiempo pantalla y sus consecuencias sobre la salud, además de comentar las consecuencias económicas personales y sociales de la insuficiente actividad física. Se comentan algunas estrategias recientes para ayudar a las personas a ser más activas físicamente. Se resume la información más reciente sobre la interacción entre actividad física regular y salud, desde la perspectiva de la función cognitiva, el cáncer, el exceso de peso, la mortalidad, las enfermedades cardiovasculares, el embarazo, las enfermedades en adultos mayores, la hipertensión, y la osteoartritis. Se reafirma la directriz general de realizar como mínimo 150 minutos de actividad física por semana de moderada a vigorosa intensidad, para una vida saludable en adultos y adultos mayores; cuando la evidencia lo permite, se presentan recomendaciones específicas.
\end{abstract}

Palabras claves: sedentarismo, vida saludable, enfermedades no contagiosas, ejercicio, bienestar.

\begin{abstract}
The purpose of this narrative review is to provide a scientifically sound basis, in Spanish, for supporting the promotion of physical activity as an indispensable component of healthy living. The review is based on two special volumes recently published in the journals Lancet and Medicine and Science in Sports and Exercise, as well as a collection of original manuscripts from other sources. The concepts of sedentary lifestyle and screen time and their health consequences are discussed, as well as the personal and socioeconomic consequences of insufficient physical activity. Some recent strategies to help people become more physically active are discussed. The latest information on the interaction between regular physical activity and health from the perspective of cognitive function, cancer, excess weight, mortality, cardiovascular disease, pregnancy, diseases in older adults, hypertension, and osteoarthritis is summarized. The general guideline of at least 150 minutes of moderate- to vigorous-intensity physical activity per week for a healthy life in adults and older persons is reaffirmed; when evidence permits, specific recommendations are presented.
\end{abstract}

Key words: sedentary lifestyle, healthy lifestyle, non-communicable diseases, exercise, wellness.

\section{Introducción}

La actividad física regular, desarrollada bajo parámetros de intensidad acordes a las condiciones de salud que presentan las personas y los objetivos planteados, es el camino indicado para adoptar la actividad física como herramienta de prevención y mejoría ante los frecuentes problemas de salud (hipertensión, diabetes, etc.) así como complemento en etapas de la vida en las mujeres como el embarazo o la función cognitiva en adultos mayores. El sedentarismo como contraposición a la actividad físi$\mathrm{ca}$, genera cargas tanto físicas como económicas inmensas que en muchos casos no pueden ser revertidas, desencadenando una muerte temprana o el pa-

Fecha recepción: 10-08-20. Fecha de aceptación: 20-04-21

Julián Camilo Garzón Mosquera

julian.garzon@ucr.ac.cr decimiento de una enfermedad.

Es difícil encontrar publicaciones formales contemporáneas en las que se refute la importancia que tiene la actividad física regular para la salud del ser humano. Al mismo tiempo, los retos laborales y la presión sobre el tiempo libre que experimentamos hoy nos plantean serios problemas para cumplir con nuestra «cuota de ejercicio» (cp. Sabean, Aragón \& Anderson, 2014, p. 19). De ahí que sea especialmente importante entender cuáles son los principales problemas asociados con la falta de actividad física, y cuáles son las soluciones que tienen más posibilidades de dar buenos resultados. Ahora bien, aunque la labor de escribir un resumen de la evidencia y pautas más recientes sobre actividad física para la salud es una tarea titánica, por el volumen de información disponible y la continua publicación de nuevos reportes, es de vital importancia ofrecer a los profesionales en ciencias del movimiento humano infor- 
mación que sea, al mismo tiempo, concreta, práctica, reciente y bien fundamentada.

El propósito de esta revisión narrativa es ofrecer una base científicamente sólida, en español, para respaldar la promoción de la actividad física como componente indispensable de una vida saludable. Para ello, se han revisado detalladamente los manuscritos en dos fuentes específicas: el suplemento de la revista LANCET del año 2016 sobre el tema (volumen 388, número 10051), y el número especial de la revista Medicine and Science in Sports and Exercise de junio de 2019 (volumen 51, número 6). En este último se presenta el informe del Comité Asesor en Actividad Física para los EE.UU. en el 2018, una actualización de la información presentada en el 2008. La información se complementa con otros manuscritos relevantes, una selección no sistemática de documentos relacionados con la actividad física, el sedentarismo y la salud, publicados entre 1996 y 2019, haciendo de este manuscrito único en la información aquí condensada, para el idioma español como una recopilación relevante en el tema de actividad física para la salud; siendo distinto en a las algunas guías actuales de actividad física para la salud como la publicada por Crespo-Salgado et al. (2015) donde se centran en recomendaciones y no en evidencia actualizada.

La primera sección de este manuscrito presenta el reto creciente de la falta de actividad física, analizando la información más reciente sobre los conceptos de tiempo sentado y tiempo pantalla — siendo estos factores que inciden sobre inactividad física—así como la carga económica que representa la inactividad física. Una segunda sección ofrece algunas pautas básicas para la promoción de la actividad física, dentro de las cuales se plantean alcances y limitaciones importantes del entrenamiento interválico de alta intensidad (en inglés, High Intensity Interval Training; HIIT), una modalidad reciente de entrenamiento que goza de mucha popularidad. En la tercera sección se presentan resúmenes sobre la interacción entre actividad física regular y salud, desde la perspectiva de nueve temas distintos: la función cognitiva, el cáncer, el exceso de peso, la mortalidad, las enfermedades cardiovasculares, el embarazo, las enfermedades en adultos mayores, la hipertensión, y la osteoartritis. Algunos de estos temas cuentan con datos más específicos y completos que otros, lo cual obedece primordialmente a la antigüedad de cada línea de investigación. Finalmente, se presenta un resumen general con algunas recomendaciones concretas.
En resumen, a lo expuesto en los párrafos anteriores introductorios para este manuscrito, es evidente el problema que aqueja la sociedad actual en cuanto a la falta de actividad física que se realiza y más aún el poco conocimiento que se tiene por parte de la población en general de las consecuencias de no tener hábitos de realizar actividad física con regularidad. Gran parte de la información científica que hay en este campo, se encuentra escrita en idioma inglés, siendo esta una barrera lingüística para la población de habla hispana y más aún una limitante importante para la divulgación de información, que podría ayudar a problemas de salud que se presentan en esta población. Así pues, el objetivo de esta revisión narrativa radica en divulgar evidencia científica actualizada principalmente publicada en dos revistas de renombre escritas en inglés, proporcionando una interpretación resumida y clara de los temas allí tratados.

\section{Metodología}

Se han seleccionado dos fuentes de información (revista $L A N C E T$ del año 2016 volumen 388, número 10051 y el número especial de la revista Medicine and Science in Sports and Exercise de junio de 2019 volumen 51, número 6), las cuales han cumplido con el criterio de tener los temas suficientes en relación con la actividad física para la salud. Asimismo, la selección de los temas allí descritos se desarrolla en este manuscrito en un orden lógico desde los problemas de no realizar actividad física para la salud hasta la evidencia más reciente de los beneficios que la actividad física trae frente a muchos problemas de salud que presenta la población. Se estableció un criterio de exclusión de la información que en estas dos publicaciones se encontraba y que no presentaba la suficiente solidez como para ser descrita dado las posibles ambigüedades que pudieran presentarse a la hora de ser interpretada. La información seleccionada, no ha sido transcrita de igual manera en que se encuentra en estas dos publicaciones, ya que se aporta un criterio de análisis propio frente a la información seleccionada y que consideramos relevante para ser difundida en la población de habla hispana.

\section{La falta de actividad física en la sociedad contemporánea}

Tiempo sentado: más allá de la poca actividad física

Originalmente, los estudios sobre «sedentarismo» 
se habían centrado en los niveles bajos de actividad física y ejercicio y sus efectos negativos sobre la morbilidad y la mortalidad, así como en las recomendaciones concretas de actividad física regular para evitar esos problemas. Más recientemente, sin embargo, se ha establecido que el pasar muchas horas diarias sentado («tiempo silla») tiene un efecto negativo sobre la salud que no necesariamente se neutraliza realizando actividad física diariamente. La acción de sentarse, bien sea en un sofá, una silla, el piso, etc. añaden al diario vivir momentos de descanso o de trabajo en muchos de los casos. Sin embargo, el estar largos períodos con las rodillas a nivel de la cadera y ocasionalmente la espalda reclinada sobre una superficie (sentado), trae consigo problemas de salud importantes como enfermedades cardiovasculares, diabetes tipo 2 y otros problemas de mortalidad. El estar largos períodos sentado o haber presentado una vida en esta posición, es un tema de estudio de muchos investigadores del área del movimiento humano y la salud. Para el estudio de tiempo sentado, la organización Sedentary Behavior Research Network (Red para la investigación de la conducta sedentaria) ratificó recientemente la definición: «(conducta sedentaria es) aquella conducta que ocurre durante la vigilia y genera un metabolismo < 1.5 MET en una posición sentada, o reclinada» (Tremblay, et al., 2017, p. 2). El término MET se utiliza para referirse a un equivalente metabólico, o el gasto energético de estar en reposo, que representa $3,5 \mathrm{~mL} \mathrm{O} / \mathrm{kg}$ min.

Chau et al. (2013) señalan que cada hora diaria adicional de estar sentado está asociada con un aumento general del 2\% del riesgo de mortalidad por todas las causas después de que se tiene en cuenta la actividad física; sin embargo, se encontró que la asociación entre el tiempo de estar sentado y el riesgo de mortalidad por todas las causas no era lineal, con un 5\% mayor riesgo de mortalidad en todas las causas por cada incremento de 1 hora en el tiempo sentado para adultos por encima de $7 \mathrm{~h} /$ día. Más aun, no hay evidencia de mayor riesgo de mortalidad por incremento de 1 hora de estar sentado en los tiempos de asiento totales diarios de 0-3 y entre 3-7 h/ día. Cuando no se hace actividad física, y se estima el total de tiempo sentado entre 4 y 8 horas y $>8$ h/ día, se asocia con un riesgo significativamente mayor de morir siendo este un $2 \%$ más y $8 \%$ más por cada incremento de 1 hora diaria ( 9 horas), respectivamente (Chau, et al., 2013). Esto sugiere que la acti- vidad física amortigua los problemas de salud asociados al tiempo de estar sentado, especialmente en la categoría más alta del «tiempo silla».

El riesgo de mortalidad aumenta progresivamente a medida que las personas pasan más tiempo sentadas durante el día (un modelo de dosis-respuesta); sin embargo, la actividad física presenta un efecto protector en personas que presentan tiempos largos de estar sentadas por actividades laborales. Según Chau y colaboradores (2013), el riesgo de mortalidad de todas las causas por pasar 10 horas al día sentado, es 34\% mayor frente a pasar sentado solamente 1 hora, en aquellas personas que hacen actividad física. Si no se realiza actividad física, el riesgo aumenta aún más: $52 \%$.

Un metaanálisis realizado con gran cuidado, en el cual se contempla una muestra acumulada mayor al millón de personas de diversas características, confirma las tendencias presentadas en el párrafo anterior (Ekelund, et al., 2016). Para aquellas personas que hacen solamente 5 min diarios de actividad física, pasar más de 8 horas al día sentadas les incrementa el riesgo de mortalidad por todas las causas en 27\%, con respecto a pasar menos de 4 horas sentadas. En cambio, para el cuartil de mayor actividad física (60$75 \mathrm{~min} /$ día), pasar más de 8 h sentado aumenta el riesgo un $4 \%$, y este aumento no es estadísticamente significativo frente a la referencia de menos de 4 «horas silla» por día. En los dos cuartiles medios de la actividad física, los niveles de mortalidad asociados con el aumento del tiempo sentado muestran la misma tendencia. En resumen, es claro que pasar muchas horas diarias sentado aumenta el riesgo de mortalidad por todas las causas; esto puede ser mitigado por la actividad física regular; sin embargo, para neutralizar el efecto negativo de las horas silla, se requiere realizar actividad física diaria. Como es mencionado por Ekelund, et al. (2016), cuanto más alto el número de horas-silla por día, tanto mayor tiene que ser la actividad física para evitar complicaciones de salud.

\section{Tiempo pantalla}

Desde mediados del siglo XX, cuando se inventó el televisor, se convirtió a través de los años en un objeto infaltable en los hogares y donde comúnmente se pasa la mayor parte del tiempo sentado o acostado frente a la pantalla. Este es un tiempo que se conoce como el «tiempo pantalla», que se ha estudiado aparte del tiempo sentado, pero está altamen- 
te relacionado con este. El tiempo empleado en la acción de ver televisión, por lo general es un tiempo de ocio que puede llegar a ser prolongado. Más aun, el tiempo pantalla se puede asociar a una falta de actividad física y se presentan muchas enfermedades no transmisibles tales como diabetes tipo 2, enfermedad cardiovascular, accidente cerebrovascular, algunos cánceres y mortalidad prematura (Ekelund et al., 2016, p. 1303). Por lo tanto, se le considera un factor potencial para muchas condiciones crónicas y de mortalidad durante la última década. Asimismo, es oportuno mencionar que en la actualidad el tiempo pantalla es considerado en todos los dispositivos tecnológicos que llevan al ser humano a permanecer frente a una pantalla (celular, video juegos, computadora, etc.).

El tiempo pantalla prolongado se asocia al riesgo creciente de presentar diabetes tipo 2, enfermedad cardiovascular, y mortalidad por todas las causas; asimismo, Ekelund et al. (2016) evidencian que, en los sujetos que presentaban un tiempo pantalla $>5 \mathrm{~h} /$ día, el peligro por mortalidad de diversas enfermedades aumenta del 16\% al 93\% frente a cuartiles de actividad establecidos. Aún para cuartiles de actividad física altos, tener un tiempo pantalla superior a $5 \mathrm{~h} /$ día se asocia significativamente con un mayor riesgo de mortalidad. En el otro extremo, los sujetos de los cuartiles de menor actividad física, que presentaban un tiempo pantalla $<1 \mathrm{~h} /$ día también presentan un mayor riesgo de mortalidad.

Kim et al. (2013) manifiestan en sus resultados que, en aquellas personas con 5 horas diarias de tiempo pantalla o más, los hombres presentan 19\% mayor probabilidad de mortalidad por todas las causas, y las mujeres un 32\%, en comparación con quienes ven la televisión menos de 1 hora diaria. Estar sentado más de 3 h/día vs. menos de 1h/día en otras actividades de ocio o durante las comidas, también se asociaron con 6-7\% y 22\% mayor riesgo de mortalidad en hombres y mujeres, respectivamente, por todas las causas; asimismo, se calcula que hay un 13\% de aumento en el riesgo de mortalidad por todas las causas por cada 2 horas de tiempo pantalla (Kim, et al., 2013).

El tiempo pantalla es un factor de riesgo a tomar en cuenta a cualquier edad. Presentar hábitos saludables de actividad física y reducir al mínimo posible los momentos en que se pasa frente a una pantalla, están relacionadas con una mejor calidad de vida y estado de salud óptimo. Aquellas personas que pre- sentan un nivel bajo de actividad física ( 5 minutos por día) aumentan la mortalidad por todas las causas en 44\% al permanecer por más de 5 horas al día de tiempo pantalla, frente a una medida de referencia de menos de una hora al día. De igual manera, el aumento de muerte por todas las causas se ve aumentado en un 15\%, a pesar de cumplir con un nivel de actividad física alto (60-75 minutos por día), al permanecer por más de 5 horas al día de tiempo pantalla, frente a la línea de referencia $<1$ hora al día (Ekelund, et al., 2016, p. 1308).

\section{La carga económica de la inactividad física}

La actividad física fue definida por la organización mundial de la salud (World Health Organization, WHO) como cualquier movimiento corporal producido por los músculos esqueléticos que requiere un gasto de energía (2020a). Así pues, la ausencia de actividad física o la inactividad física se ha convertido en un factor común de la sociedad actual, siendo reconocido como una de las principales causas de los problemas de salud pública que viven las comunidades. La inactividad física se asocia con múltiples enfermedades crónicas y muertes tempranas. Asimismo, el punto económico de la inactividad física es de magnitudes alarmantes para las personas que, por no tener hábitos adecuados de salud en cuanto a la actividad física, padecen un gasto innecesario y que se prolonga por el resto de la vida. El costo no es solamente individual: la sociedad como tal paga un precio sumamente alto por la inactividad física de sus miembros.

La información que se presenta a continuación está fundamentada en el manuscrito de Ding et al. (2016a). Estos autores señalan que el costo de la inactividad física podría estar, para una persona, en el rango del 1 al 4\% de los costos totales de atención directa de la salud, teniendo en cuenta que estas cifras estimadas son para países donde se presentan ingresos altos. A su vez, los autores analizan valores de 142 países que representan el 93\% de la población mundial, con los cuales se logra estimar que la inactividad física representó, para el año 2013, alrededor de 67.5 millardos (miles de millones) de dólares, analizando los gastos de la salud pública y las pérdidas en productividad. Este valor equivale al PIB total de un país como Costa Rica, país que ocupó el puesto 80 de 193 países para ese año (FMI, 2013). Asimismo, los costos mundiales de atender las enfermedades consecuencia de la inactividad física, en 
el año 2013, llegaron a representar el 63\% de los gastos sanitarios (Ding, et al., 2016a).

Los pagos de individuos y hogares en regiones como Latinoamérica o el sudeste asiático, evidencian un ahogamiento financiero por los gastos médicos que deben realizar, esto también ocasionado por la poca o limitada estructura de salud pública de estas regiones; generalmente en los países con menores recursos o pobres, mayor es la insatisfacción de las necesidades de salud pública de la población y es donde son más altos los índices de morbilidad y mortalidad temprana. Además, al existir niveles de actividad física más altos en los países de altos ingresos, eso contribuye a tener una carga económica menor que la representada en países de bajos a medianos ingresos.

Es importante para los gobiernos de países tanto de altos como de medianos y bajos ingresos, implementar programas de promoción de la actividad física en todos los rangos de edades y actividades, para disminuir todos los gastos innecesarios por la inactividad física, a su vez mejorando los índices económicos por individuo o por hogar y a nivel global disminuir los índices de muertes por la inactividad. Los datos que respaldan esta recomendación se basan en los costos de la inactividad física, específicamente en la región latinoamericana, donde ascienden a \$3.2 millardos de dólares americanos anuales, lo cual representa el 53\% de los costos directos de la salud pública en los países de esta región.

\section{Estrategias para promover la actividad físi-} ca

Promoción de la actividad física: las pautas básicas

Se ha identificado que la actividad física proporciona un sinnúmero de beneficios, desde reducir al mínimo los efectos de la ansiedad y la depresión, hasta mejorar la calidad del sueño; asimismo, reducir los riesgos de desarrollar diabetes, enfermedades cardíacas, cáncer, muerte prematura, etc. El conocimiento y los beneficios son claros y se han divulgado a través de los años; sin embargo, gran parte de la población mundial aún no se concientiza o no sabe qué hacer para producir los beneficios expuestos por hacer actividad física. Para el año 2015, se conocía que poco menos de la mitad de los estadounidenses llegaban a cumplir con las pautas según la edad para una actividad física aeróbica; asimismo, un tercio de los adultos informaron estar inactivos durante su tiem- po libre (Physical Activity Healthy People 2020, s. f.). Además, países latinoamericanos (Brasil, Argentina) presentan valores mayores de inactividad física $(>$ $50 \%$ ) en población mayor de 15 años (Hallal, et al.2012) y a nivel global Guthold et al. (2018) pudieron determinar que, la insuficiencia de actividad física estaba en un $27,5 \%$ de la población, esto bajo una muestra hecha en 168 países.

Aparte de divulgar el conocimiento, que quizás es más importante para los profesionales en ciencias del movimiento humano, es crucial utilizar estrategias efectivas que le ayuden a la población. La promoción de la actividad física desde mediados del siglo XX, se ha enfocado en intervenciones a nivel individual que consisten en programas de persona a persona o de pequeños grupos, buscando los mejores resultados para el objetivo final de realizar actividad física, siempre intentando seguir las bases científicas de estudios experimentales que presenten resultados favorables ante la prevención de las enfermedades y cambios al comportamiento sedentario. Existen diversas técnicas que ayudan al cambio de comportamientos con miras a iniciar un hábito para realizar actividad física: la auto supervisión del comportamiento y la formación de la intención, son técnicas con mayores resultados favorables. Asimismo, proporcionar recompensas por asistencia y la comprensión de efectos al lograr metas de actividad física en una variedad de grupos de edad, se plantean como caminos correctos a elegir para presentar cambios al comportamiento sedentario (King et al., 2019).

Las intervenciones efectivas que se han reportado en sujetos jóvenes, se han basado en la educación personal y actividades experienciales que han incluido a la familia en el programa de actividad física a realizar (Cushing, et al., 2014). Como ejemplo de intervenciones que seguirían esta línea efectiva de promoción de la salud en jóvenes, serían los ejercicios supervisados, la danza y las actividades deportivas.

Algunos avances tecnológicos han facilitado la promoción de la actividad física, en particular los dispositivos llamados «wearables», los cuales son dispositivos inteligentes que uno usa como parte de su vestimenta regular, especialmente los relojes con monitores de frecuencia cardíaca o contadores de pasos, o los celulares inteligentes. El monitoreo de la cantidad de pasos diarios de cada persona ha permitido registrar objetivamente (aunque con precisión aproximada) la actividad física diaria, resultan- 
do en la publicación de varios estudios longitudinales que muestran una relación inversa entre los pasos diarios y la morbilidad, así como entre los pasos diarios y el riesgo de muerte (Kraus, et al., 2019). Esos estudios también han permitido establecer metas diarias de actividad física; la meta más popular, con sustento científico limitado, es la de cumplir con un mínimo de diez mil pasos diarios, aunque se considera que hace falta más investigación para poder traducir esto en una recomendación sólida de salud pública (Kraus, et al. 2019). De igual manera la utilización de aplicaciones móviles, han logrado mejorar y aumentar la práctica deportiva (Díaz et al., 2019)

Los adultos mayores son una población que presenta características particulares, ya que muchos de estos ya traen consigo comportamientos sedentarios de muchos años y, más aún, los problemas de salud, que por la edad son más notorios a la hora de establecer una estrategia para iniciar una actividad física que ayude a mejorar sus días. Se ha demostrado que las intervenciones dirigidas específicamente a las necesidades y gustos, son eficaces para promover el aumento de la actividad física, siempre y cuando sean en periodos por lo menos de un año (Physical Activity Guidelines Advisory Committee, 2018). Se ha informado que los tipos de estrategias eficaces para adultos mayores son: el asesoramiento tanto individual como grupal, la resolución de problemas en torno a las barreras para la actividad física, el apoyo social, el modelado con demostraciones similares de las actividades físicas a realizar y la utilización de recompensas vinculadas al cambio de comportamiento (French, Olander, Chisholm, \& Mc Sharry, 2014).

Dentro de las estrategias de recompensa con adultos mayores, un ejemplo evidenciado en un metaanálisis mostró el efecto positivo de proporcionar lotería y aumentar los incentivos por la asistencia a las sesiones de ejercicio en comparación con ningún incentivo. Para intervenciones de corta duración que durarían de 4 a 26 semanas, los resultados evidenciaron un aumento en la asistencia al ejercicio del 11,55\% (Mitchell, et al., 2013); de igual manera, Mitchell et al. (2013) realizaron un metaanálisis donde se incluyeron más de 6000 participantes y donde se pudo evidenciar que los incentivos financieros lograron un aumento en la cantidad de pasos que los adultos mayores realizaban tanto durante como postintervención.

Es importante entender que incluso los pequeños efectos de una intervención pueden traducirse en impactos significativos en la salud y así lograr ser promovida eficazmente a un gran segmento de la población.

En el ámbito escolar, las intervenciones denominadas multicomponente, tienden a ser las más eficaces. Entre ellas está «CATCH» (Luepker, et al., 1996) o «SPARK» (Sallis, et al., 1997); estas intervenciones o programas incluyen cambios estructurales en las clases de educación física, descansos activos entre clases, sesiones de actividades pre y post jornada escolar, transporte activo hacia y desde la escuela ( p. ej. bicicleta o caminando), desarrollar habilidades conductuales que promuevan la participación en actividades físicas; asimismo, presentar los recursos materiales necesarios para estas actividades. Se ha evidenciado que estas intervenciones aumentan significativamente la actividad física durante las horas escolares. Diversos estudios demuestran que la implementación de un plan de estudios de educación física bien diseñado con maestros debidamente capacitados mejorará la cantidad de actividad física entre moderada a vigorosa. Un ejemplo es expuesto por Lonsdale et al. (2013), los cuales reportan un aumento del $24 \%$ en el tiempo de aprendizaje activo durante la clase de educación física, frente a los participantes del grupo control, independientemente de la edad, el sexo y la duración de la intervención (Lonsdale, et al., 2013). Asimismo, van de Kop et al. (2019, p. 192) describen en su metaanálisis los pequeños pero significativos beneficios que se logran obtener mediante intervenciones multicomponente.

\section{Entrenamiento HIIT}

Muchísimos programas de actividad física para la salud se han centrado en el ejercicio de baja o moderada intensidad y de larga distancia o duración. Más recientemente, el entrenamiento interválico de alta intensidad, conocido comúnmente como HIIT (del inglés high intensity interval training), está enfocado en trabajos de corta duración con énfasis en la resistencia aeróbica de quien los realiza. Este tipo de entrenamiento, popular en gran parte de la población que realiza actividad física, se caracteriza por su flexibilidad para adaptarse a distintos ambientes en donde se ejecuten actividades físicas. Un ejemplo de la flexibilidad de este tipo de entrenamiento se ha observado en la población que realiza actividad física por salud, donde HIIT ha logrado presentar resultados evidentes de pérdida de grasa corporal, estando siempre rivalizado por el entrenamiento tradicio- 
nal, que a menudo se realiza también con el mismo fin.

La «alta intensidad» de los entrenamientos, puede presentar un nivel mínimo de 65\% de la reserva cardiaca máxima o estar sobre un $60 \%$ de $\mathrm{VO}^{2} \max$ (consumo máximo de oxígeno) o tan alto como el esfuerzo realizado por una carrera de corta distancia (menor a 50 metros) a máxima velocidad; al cumplir con los lineamientos establecidos para considerarse HIIT, se obtendrán repuestas al ejercicio tanto desde un punto de vista cardiopulmonar, como metabólico o neuromuscular. Una muestra de esto es el estudio de Sloth et al. (2013), en el cual se establece que el ejercicio interválico de alta intensidad, ejecutado durante un periodo de 4 a 8 semanas, logra aumentar los valores de $\mathrm{VO}^{2}$ max entre 4 y 15\%, tanto en personas no entrenadas como en personas físicamente activas (Sloth, Sloth, Overgaard, \& Dalgas, 2013).

Alguna evidencia reciente (Batacan, Duncan, Dalbo, Tucker, \& Fenning, 2017; Jelleyman, et al. 2015; Kessler, Sisson, \& Short, 2012), ha demostrado la reducción de grasa corporal y de la presión arterial en personas adultas que presentaban sobrepeso, quienes tuvieron sesiones de entrenamiento HIIT en períodos cortos-medianos de tiempo. Estos resultados importantes sobre la salud se obtuvieron tanto en hombres como mujeres. Estas mejoras inducidas por HIIT en los factores de riesgo de enfermedades cardiometabólicas son comparables con las que resultan del ejercicio aeróbico continuo de intensidad moderada, y es más probable que ocurran en adultos con mayor riesgo de enfermedades cardiovasculares y diabetes, en comparación con adultos sanos. Más aun, es importante resaltar que los adultos clasificados con sobrepeso u obesidad llegan a responder mejor que los adultos con peso normal a los efectos de HIIT para la reducción de la presión arterial y la mejoría en la composición corporal. Asimismo, Espinoza-Salinas et al. (2020) manifiestan los beneficios obtenidos después de un protocolo interválico de alta intensidad sobre la actividad cardiovagal en personas con sobrepeso, aumentando la modulación parasimpática en reposo.

La gran cantidad de protocolos e intensidades que se presentan como HIIT, por su variedad, pueden llegar a tornarse confusas en la práctica. Sin embargo, existe una clasificación general basada en la intensidad del ejercicio con dos modalidades, primeramente, se presentan los entrenamientos interválicos de carrera o sprint interval training (SIT), caracterizados por intensidades superiores al $\mathrm{VO}^{2}$ max y la intensidad vigorosa aeróbica o vigorous aerobic intensity, caracterizándose por estar en el rango de 64 - 90\% del $\mathrm{VO}^{2}$ max, clasificación resumida por Campbell et al. (2019). Al hacer los análisis conforme a esta clasificación, no parece que el HIIT haya demostrado una mayor efectividad que el entrenamiento continuo a una intensidad moderada, para la pérdida de grasa corporal en personas obesas o con sobrepeso. Sin embargo, la menor cantidad de tiempo requerido para ejecutar sesiones de HIIT, proporciona ventajas en la mejora de la potencia aeróbica y algunos marcadores de salud, llegando a considerar esta modalidad de entrenamiento como una muy buena opción para realizar actividad física (Campbell, et al., 2019).

Una receta evidenciada por Maillard et al. (2018), puntualiza que: se debe alternar la alta intensidad (4 minutos) con la recuperación activa (3 minutos), siendo entrenamientos enfocados en actividades deportivas de carrera o ciclismo, esperando tener una duración mínima de 12 semanas con tres sesiones por semana y lo más importante es alcanzar un nivel e» 90\% de la frecuencia cardiaca máxima, durante los momentos de alta intensidad (Maillard, et al., 2018). Siempre y cuando los sujetos que lleven la actividad física a esta alta intensidad tengan en cuenta las recomendaciones pertinentes de cautela. A pesar de que el HIIT con una alta intensidad de trabajo resulta beneficioso sobre la capacidad aeróbica, también puede conducir a un aumento en el riesgo de eventos cardíacos adversos: Bizzozero y Díaz (2019) indican que el entrenamiento tipo «HIIT debe realizarse con aprobación médica, en pacientes estables, con la supervisión de un profesional del ejercicio y con una progresión adecuada» (p. 22). De igual manera, se hace alusión a este tipo de ejercicio en este manuscrito al observar la tendencia actual y como ha sido tema de conversación científica en los últimos años en cuanto a su eficiencia en diversos aspectos físicos y de población, entendiendo los temas psicológicos motivacionales y de adherencia que no son los más positivos en población con características de inactividad física, sobrepeso, obesidad, etc. (Kinnafick et al., 2018)

\section{Interacción entre actividad física y salud}

Actividad física y enfermedades cardiovasculares

Las enfermedades cardiovasculares son uno de los mayores problemas de salud pública a nivel mundial. 
La asociación americana del corazón (American Heart Association, AHA) evidencio por medio de Benjamin et al. (2017) señala en su resumen estadistico que, en Estados Unidos, ocurren más de 801.000 muertes anuales por enfermedad cardiovascular. Ese número equivale a una de cada tres muertes en Estados Unidos; Asimismo, Las enfermedades cardiovasculares cobran más vidas que todos los tipos de cáncer y enfermedad crónica de vías respiratorias inferiores combinados. La enfermedad cardiovascular es la causa principal de mortalidad a nivel mundial, y en 2013 causó más de 17.3 millones de muertes, una cifra que se proyecta aumentará a más de 23.6 millones para el año 2030 (Benjamin, et al., 2017). Por otra parte, la Organización Panamericana de la Salud (OPS) y la Organización Mundial de la salud (OMS) informaron que, para el año 2011 en Costa Rica, del total de mortalidad prematura por todas las causas (4465 muertes), las enfermedades cardiovasculares representaron el $27 \%$ en hombres entre 30-69 años. Para mujeres en el mismo rango de edad, con un total de mortalidad prematura por todas las causas de 2565, las muertes por enfermedades cardiovasculares representaron el 24\% (Organización Panamericana de la Salud (OPS) / Organización Mundial de la Salud (OMS), 2014).

Las enfermedades cardiovasculares son un problema que aqueja a una gran parte de la población mundial; al mismo tiempo, se ha evidenciado una relación inversa entre la realización de actividad física y las causas de mortalidad debidas a problemas cardiovasculares (Kraus, et al., 2019). La afirmación anterior es confirmada por Sattelmair et al. (2011), quienes evidencian una reducción de 14\% en la aparición de enfermedades coronarias, al realizar actividad física de intensidad moderada a vigorosa, presentando una disminución temprana del riesgo. Por ejemplo, para una persona de $70 \mathrm{Kg}$. que presenta una intensidad de actividad baja, requiere un volumen suficientemente alto para lograr un gasto de $600 \mathrm{Kcal} *$ semana (Sattelmair, et al., 2011).

Realizar actividad física a un volumen semanal de 500 MET-min determina una reducción de riesgo para enfermedades cardiovasculares de un 33\% en comparación con personas sedentarias (Pandey, et al., 2015); asimismo, se evidencia la relación inversa entre la intensidad y el tiempo destinado a la actividad física, siendo este estadísticamente significativo. Igualmente, Sattelmair et al. (2011) manifiestan que realizar 150 minutos por semana de actividad física logra disminuir el riesgo de desarrollar enfermedades cardiovasculares en un 14\%, más aún, al realizar el doble de actividad física (300 minutos por semana) se reduce el riesgo en un 20\%. Resumiendo, 150 minutos semanales a intensidad de moderada a vigorosa es suficiente para obtener beneficios cardiovasculares significativos, mientras que cantidades mayores proporcionan beneficios adicionales.

\section{Actividad física e hipertensión}

En los Estados Unidos, una de cada tres personas muere por enfermedades cardiovasculares; a nivel mundial este tipo de muerte también representa el 33\% (Pescatello, et al., 2019). La hipertensión es el factor de riesgo que se presenta con mayor frecuencia, a su vez, es considerado el de mayor costo. Tomando en cuenta los umbrales establecidos por el séptimo comité nacional conjunto (the Joint National Committee Seven, JNC 7) se predice que existe un 90\% de probabilidad de padecer de hipertensión durante el tiempo de vida y que una de cada cinco personas padecerá de prehipertensión dentro de los próximos cuatro años (Chobanian, 2003). El Colegio de Cardiología de los EE.UU. (American College of Cardiology, ACC) y la Asociación del Corazón de los EE.UU. (American Heart Association, AHA), redefinieron recientemente la hipertensión a un umbral menor de presión sanguínea sistólica (PAS) de $130 \mathrm{~mm} \mathrm{Hg} \mathrm{y} 80 \mathrm{~mm} \mathrm{Hg}$ para presión sanguínea diastólica (PAD). Este cambio ahora clasifica casi a la mitad de los adultos estadounidenses con hipertensión (Gijón-Conde et al., 2018). En Costa Rica el 34\% de la población padece de hipertensión, según datos del año 2015 (Hospital Clínica Bíblica, 2015), convirtiendo a esta enfermedad en un problema de salud pública que cada año se ve en aumento por la falta de concientización y programas de prevención.

Liu et al. (2017) tomaron una amplia muestra de 330.222 adultos que presentaban una presión sanguínea normal, con un seguimiento que fue de los 2 a 20 años. Durante este tiempo se evidenciaron 67.698 casos de hipertensión, mostrando una relación de dosis-respuesta a la actividad física con resultados significativos: bajo la perspectiva de realizar actividades que estén en los 10 MET-h por semana, se obtuvo una reducción del 6\% en el riesgo de desarrollar hipertensión; asimismo, el efecto protector subió un $6 \%$ por cada aumento adicional de 10

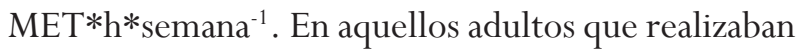
$20 \mathrm{MET}^{*} \mathrm{~h} *$ semana $^{-1}$ de actividad física ligera, mo- 
derada y/o vigorosa, el riesgo de hipertensión se redujo en un $12 \%$, más aún, los que realizaron actividad física ligera, moderada y/o vigorosa de 60 MET*h* ${ }^{*}$ semana $^{-1}$, el riesgo de hipertensión se redujo en un 33\%. La evidencia sólida demuestra una relación inversa entre la actividad física y la hipertensión incidente en adultos con presión sanguínea normal (Liu, et al., 2017).

La magnitud de las reducciones de presión sanguínea osciló entre 5-17 mm Hg para la PAS y de 2 a $10 \mathrm{~mm} \mathrm{Hg}$ para PAD. La evidencia sólida demuestra que la actividad física reduce la presión arterial entre los adultos con hipertensión, asimismo, Law et al. (2009) señalan que las mayores reducciones de presión sanguínea bajo un programa de actividad física, se logran observar en personas con hipertensión (5 $\mathrm{mm} \mathrm{Hg}$ a $8 \mathrm{~mm} \mathrm{Hg}, 4 \%$ a 6\% del nivel de presión sanguínea en reposo), seguido de personas con prehipertensión (2 a 4 mm, 2\% a 4\% de presión sanguínea en reposo) (Law, Morris, \& Wald, 2009). Adicionalmente, Pescatello et al. (2019) concluyen que la relación entre el nivel de presión sanguínea en reposo y la respuesta de la presión sanguínea a la actividad física, no varía según el tipo de actividad (aeróbico, resistencia dinámica, combinado) entre adultos con presión arterial normal, prehipertensión e hipertensión.

\section{Actividad física y mortalidad prematura}

La Organización Mundial de la Salud (OMS), señala que la falta de actividad física representa el 6\% de las muertes a nivel mundial, ocupando el cuarto lugar precedido por la hipertensión (13\%), consumo de tabaco $(9 \%)$ y diabetes (6\%). Asimismo, la inactividad física representa la principal causa de aproximadamente el 21-25\% de los cánceres de mama y de colon, $27 \%$ de la diabetes y un 30\% de las cardiopatías isquémicas (Cigarroa, Sarqui, \& Lamana, 2016). También se estima que aproximadamente 3,2 millones de personas mueren a causa del sedentarismo cada año. Las personas con poca actividad física corren un riesgo entre un $20 \%$ y un $30 \%$ mayor que las otras de morir prematuramente por cualquier causa (Alwan, et al., 2011).

Realizar actividad física siguiendo las directrices recomendadas por la guía del comité asesor en actividad física para los EE.UU. (Physical Activity Guidelines Advisory Committee, 2018), en la cual se recomienda tener un tiempo de 150-300 min*semana a intensidad moderada o 75-150 min*semana de actividad vigorosa, representa el tiempo e intensidad suficientes para presentar una condición física aceptable, asimismo, muestran una asociación inversa a la mortalidad.

Las actividades físicas comunes de montar en bicicleta y caminar son actividades recomendadas por muchos educadores físicos para iniciar un hábito saludable. La actividad de bicicleta presenta la característica de tener un impacto menor en los músculos y la actividad de caminata la característica de ser una acción natural y sin mayor complicación para gran parte de la población. Kelly et al. (2014) señalan que realizar bicicleta o caminata proporcionan similares beneficios a la salud, así como una reducción de riesgo por mortalidad en un $11 \%$ y $10 \%$ respectivamente (Kelly, et al., 2014). Estas actividades se deben ejecutar bajo lineamientos profesionales, buscando estar en una zona de intensidad moderada, siendo esta la intensidad donde mayores beneficios se han encontrado.

Aquellos adultos mayores que presentan un deterioro natural en su equilibrio, eligen realizar la actividad de caminata como alternativa de actividad física. Diversos autores han estudiado y analizado la marcha mediante metaanálisis. Entre los destacables se encuentran Hamer y Chida (2008) quienes asocian la caminata con beneficios importantes a la salud y a la reducción de mortalidad; lo anterior se logra con una intensidad moderada de $3.2 \mathrm{~km} * \mathrm{hr}$ y un promedio de 5 horas de caminata por semana (Hamer \& chida, 2008). Asimismo, Kraus et al. (2019) resaltan que seguir un plan de actividad física constante a intensidad moderada a vigorosa, sin importar la disciplina a realizar, ha logrado llegar a presentar hasta un 75\% de reducción de riesgo en la mortalidad temprana.

\section{Actividad fisica y embarazo}

La actividad física proporciona beneficios a la salud indistintamente del género, pero en el caso específico de la mujer, en su ciclo de vida por lo general presenta una o más etapas de embarazo, durante las cuales se experimentan múltiples cambios hormonales, fisiológicos y biomecánicos, como lo son el aumento del volumen de sangre y ritmo cardíaco, aumento de peso y cambio en el centro de masa. Existen varios temas centrales relacionados con la actividad física durante el embarazo: cuáles son los riesgos de ejercitarse en esta etapa, particularmente para la salud del feto y cuáles los posibles benefi- 
cios de mantenerse físicamente activa. Entre estos últimos, interesa en particular la prevención de la ganancia excesiva de peso en la madre y el bebé, la prevención de la diabetes y de la hipertensión gestacional de la madre, la posibilidad de disminuir los partos por cesárea y la macrosomía fetal (peso de más de $4 \mathrm{Kg}$. al momento de nacer).

Se han establecido lineamientos para esta población por parte del el Colegio Americano de Obstetras y Ginecólogos, (American College of Obstetricians and Gynecologists, ACOGý), entre los cuales resalta el realizar actividad física a una intensidad moderada durante 20-30 min*día ${ }^{-1}$ (Artal, 2003), siendo reafirmado este lineamiento en su publicación más reciente (American College of Obstetricians and Gynecologists, 2017); asimismo, la organización mundial de la salud (World Health Organization, WHO) señala en su informe más actual, la realización de actividad física aeróbica para mujeres en embarazo de al menos 3 veces por semana y con una duración que oscile entre 30-60 minutos (2020b, p. 49). El realizar actividad física dentro del rango establecido y siguiendo las recomendaciones para las condiciones y etapas del embarazo, logra prevenir o disminuir el riego de presentar diabetes, hipertensión gestacional, debilitación fetal en el crecimiento asociada a enfermedades cardiovasculares, y mortalidad temprana en la madre (Rich-Edwards, Fraser, Lawlor, \& Catov, 2013).

Durante el periodo de gestación, es persistente la inactividad física de la madre, generando una serie de complicaciones tanto para la mujer como para el feto. Evenson et al. (2014) resaltan la ganancia de peso excesiva durante el embarazo y las consecuencias postparto de obesidad. Un ejemplo se muestra en las cifras reportadas por la encuesta nacional de salud y nutrición de los estados unidos (The National Health and Nutrition Survey) entre 2007-2014, donde solo entre 23 y 29\% de las mujeres embarazadas estadounidenses cumplieron con las pautas mínimas de actividad física (Hesketh \& Evenson, 2016). Asimismo, muchas mujeres que realizan actividad física constante, al quedar en embarazo han reportado una disminución o pausa completa de la actividad física que venían realizando, tanto durante el período de gestación como postparto. Esa interrupción es una lástima, ya que un análisis metaanálítico (da Silva, Ricardo, Evenson, \& Hallal, 2017) en el cual se comparó a dos grupos de mujeres en gestación (actividad física y control estándar / solo control estándar), reportó que el grupo que realizó actividad física durante el periodo de gestación obtuvo una ganancia menor que el grupo control estándar: -1,11 kg (IC 95\% - 1,59-0,69), evidenciando los resultados positivos para la salud de la madre postparto en la ganancia natural de peso que pueda presentar y que logra ser controlada sin llegar a una obesidad postparto.

El cumplimiento de los lineamientos del Comité Asesor de Pautas de Actividad Física (en inglés, Physical Activity Guidelines Advisory Committee) (2018) de $150 \mathrm{~min} *$ semana de actividad física durante el embarazo, produjo una menor ganancia de peso, viéndose atenuada la ganancia de peso gestacional que evidentemente debe ocurrir durante este periodo. También se presenta un menor riesgo en el aumento de peso posparto, obesidad futura y nacimiento de un bebé con macrosomía (Deputy, Sharma, \& Kim, 2015).

La diabetes mellitus gestacional (DMG) se presenta en 5-9\% de las mujeres embarazadas. Esto produce problemas no solamente para la madre por su riesgo aumentado de diabetes tipo 2 posparto y mayor probabilidad de tener un parto por cesárea, sino para el bebé, que tiene mayor probabilidad de macrosomía o padecer de hipoglucemia neonatal. El riesgo de DMG se puede reducir entre 25 y 30\% realizando actividad física moderada, frente a las mujeres inactivas (DeSisto, Kim, \& Sharma, 2014).

En relación con los riesgos del ejercicio en la etapa de embarazo, es importante evitar actividades de contacto (taekwondo, boxeo, judo) y de gran impacto o alto riesgo de caída (bicicleta, patinaje), ya que se podría presentar desprendimiento de placenta. También, es importante evitar actividades con cambios bruscos de velocidad o dirección, por el riesgo que representa la mayor laxitud de ligamentos presente durante el embarazo (Del Castillo, 2011).

Actividad física y enfermedades en adultos mayores

El número de adultos mayores en la población actual es cada día mayor, un ejemplo de este aumento considerable se observa en la población adulta mayor ( $>65$ años) estadounidense que a partir del 2016 representaba el 13\% del total y que para el 2030 se prevé llegará al 19\% (Dipietro, et al., 2019), llegando a duplicar el aumento que se presentó para el año 2000. Asimismo, el Instituto Nacional de Estadística y Censos (INEC) de Costa Rica señala que actualmente la población adulta mayor (65 años o más) representa $8,2 \%$ del total de la población y 
para 2048 se proyecta que el porcentaje aumente al 20\%, mientras que en 1988 era de 4,7\%. Por consiguiente, la prevención de enfermedades crónicas, el monitoreo de la condición funcional y la conservación de la independencia física para la vejez representan un gran desafío para la salud. Esto es particularmente problemático a la luz de la poca actividad física en este grupo etario. Por ejemplo, en los EE.UU. existe un bajo porcentaje (27\%) de adultos mayores que siguen los lineamientos de actividad física recomendados para presentar una condición física adecuada (Lee, et al., 2012). Si se asumiera una proporción similar en la población latinoamericana, es obvio que es de suma importancia concientizar y aumentar la participación en la actividad física regular.

Las diversas actividades a realizar están clasificadas según la función principal a cumplir, como lo son la fuerza, la velocidad de marcha, el equilibrio o los ejercicios funcionales relacionados con el diario vivir, presentando una resistencia progresiva a la actividad con ejercicios multicomponente, videojuegos, taichí, yoga o baile. Los ejercicios multicomponente (combinaciones de aeróbico, fortalecimiento muscular, equilibrio y flexibilidad); han logrado reducir significativamente el riesgo de lesiones relacionadas con caídas entre un 32-40\%, incluyendo caídas graves con resultados de fracturas óseas, traumatismo craneoencefálico, lesión de tejido blando de herida abierta, o cualquier otra lesión que requiera atención médica o ingreso al hospital (El-Khoury, Cassou, Charles, \& Dargent-Molina, 2013; Secretariat, 2008). Asimismo, hay una reducción en el riesgo de caídas en entornos comunitarios y domésticos del 40-66\%, gracias al ejercicio multicomponente en adultos mayores; quienes padecen de la enfermedad de Parkinson, también han experimentado beneficios a su condición con ejercicio multicomponente (Cauley, et al., 2013).

Aquellos programas de actividad física que contemplan el equilibrio, la fuerza, la resistencia, o la caminata son eficaces para reducir el riesgo de lesiones y fracturas relacionadas con caídas en adultos mayores, siendo programas relevantes para mejorar la salud pública en la vejez, dada la alta prevalencia de lesiones y fracturas relacionadas con caídas en esta población, así como la consecuente morbilidad, discapacidad y reducción de la calidad de vida. Por consiguiente, realizar actividad física en estas edades, también beneficiará en reducir los gastos médicos generados comúnmente en esta población, como lo señala el consejo nacional de envejecimiento ( en inglés, National Council on Aging, NCOA) el cual señala que cerca del $80 \%$ de los adultos de 60 años de edad tienen al menos una afección crónica y el 77\% tienen al menos dos, también, aproximadamente el 20-30\% de los adultos mayores de 65 años sufren de deterioro cognitivo leve o demencia (2015). Estos problemas se pueden ver reducidos o mitigados realizando actividades físicas a intensidades moderadas, controlando los parámetros de salud correspondientes a las guías internacionales (Physical Activity Guidelines Advisory Committee, 2018).

\section{Actividad física y cáncer}

El cáncer es una enfermedad que se manifiesta en distintas partes del cuerpo humano y, para muchas personas, es sinónimo de muerte, tragedia, desesperanza. Esta enfermedad afecta a gran parte de la población, llegando a ser diagnosticada en algunos casos desde etapas muy tempranas, donde los tratamientos presentan un gran porcentaje de efectividad para ser eliminado, o en etapas terminales, en las cuales los tratamientos poco o nada pueden hacer para salvar la vida. Según estadísticas del Centro Internacional de Investigaciones sobre el Cáncer (International Agency for Research on Cancer, 2018), se esperaba que para 2018 hubiera alrededor de 18 millones de nuevos casos de cáncer, de los cuales la mitad no podrían ser curados, ocasionando la muerte de los sujetos que los padecieran. Sin embargo, se espera que el número de sobrevivientes de cáncer supere los 20 millones para 2026 (Miller, et al., 2016).

La actividad física se ha asociado a la prevención de diversas enfermedades como: riesgo cardiovascular, diabetes, osteoporosis, obesidad y algunas enfermedades mentales. También se asocia la actividad física, con una mejor calidad de vida para quienes ya están enfermos y, aun así, la realizan de forma habitual. Según lo afirman López-Köstner y Zarate (2012), la desregulación de las cascadas inflamatorias, está asociada con la aparición de diversos tipos de cáncer (pulmón, colorectal, gástrico, páncreas); en esta línea, se ha observado que realizar actividad física ayuda a reducir dichas cascadas mediante el control de la inflamación crónica. Asimismo, se dice que los efectos de la actividad física se presentan a nivel sistémico, al aumentar la sensibilidad a la insulina, además de disminuir la inflamación crónica. Aunque el cáncer puede tener distintas causas, como factores genéticos, factores ambienta- 
les, el estilo de vida o la interacción entre estos (Cogliano, et al., 2011), se hace necesario generar programas de prevención primaria y secundaria. Estos programas se enfocan mayoritariamente en los cambios en el estilo de vida, basándose en décadas de estudios científicos que identifican a un estilo de vida físicamente activa como una herramienta protectora (McTiernan, et al., 2019). La actividad física regular puede, por lo tanto, tener dos efectos positivos sobre el cáncer (Keimling et al., 2014; Behrens et al., 2014; Schmid et al., 2015; Liu et al., 2016): como mecanismo de prevención primaria, y como una manera de mejorar el estilo de vida de las personas que ya lo padecen al mismo tiempo que se evita la progresión rápida de la enfermedad como prevención secundaria (Keimling et al., 2014; Behrens et al., 2014; Schmid et al., 2015; Liu et al., 2016).

Los estudios científicos que han tratado el efecto de la actividad física frente a diversos tipos de cáncer, presentan mediciones heterogéneas de la actividad física; sin embargo, en general se incluyen actividades como caminar, correr y montar bicicleta. Estas actividades son simples y se pueden ejecutar a intensidad moderada, y gracias a ellas se ha podido evidenciar una reducción de riesgo importante en cáncer: vejiga (15\%), mama (12-21\%), colon (19\%), endometrio (20\%), esófago (21\%), gástrico $(19 \%)$, renal (12\%), en comparación con cáncer de ovario, próstata, páncreas, tiroides, donde la evidencia es muy limitada y sin reducciones reportadas (McTiernan, et al., 2019). Asimismo, se ha intentado establecer la dosis-respuesta de la actividad física frente a los diversos tipos de cáncer, pero no hay un consenso de la cantidad o la clase de actividad física a realizar, debido a la poca rigurosidad metodológica de los estudios. Sin embargo, se logra consolidar que los resultados más favorables se han identificado en el cáncer de vejiga, mama, colon, endometrio y gástrico. Esto ha sido comprobado por de Rezende et al. (2018) quienes asocian la actividad física con menor riesgo de mortalidad para varios tipos cáncer; sin embargo, solo las asociaciones de cáncer de colon y de mama, presentaron el mayor respaldo estadistico en cuanto a un menor riego.

Desde el momento en que es diagnosticado un cáncer, las personas inician una nueva etapa en la vida, donde se espera que remplacen los hábitos negativos (p. ej., sedentarismo) y se adopten hábitos positivos (p. ej., actividad física, alimentación); estos cambios han sido estudiados y reportados a la comunidad con diversos tipos de cáncer (mama, colorectal, próstata). Esta prevención secundaria ha llegado a presentar reducciones significativas en el riesgo de mortalidad de $48 \%$ y $42 \%$ para los cánceres de mama y colorectal respectivamente y 37\% - 49\% para el cáncer de próstata, todo con la implementación de actividad física realizada a las capacidades de cada persona según la etapa en que se encuentre (McTiernan, et al., 2019). Bonn et al. (2015) señalan que, en población diagnosticada con cáncer de próstata, realizar caminatas e» 20 min por día y con una frecuencia mínima de tres veces por semana, se logra tener una reducción significativa de la mortalidad especifica por este cáncer.

En resumen, la relevancia que presenta la actividad física sobre una persona que ha sido diagnosticada con un cáncer, tanto en la calidad de vida, como en la reducción de riesgo, la estabilidad financiera y la mortalidad, demuestra la importancia de este factor. Pareciera que la recomendación principal es realizar actividad física según la capacidad de cada persona. De ser posible, la actividad deberá ser de tipo aeróbico, un mínimo de 20 min por día, tres veces o más por semana.

\section{Actividad fisica y peso}

Presentar un exceso de peso corporal es un factor de riesgo importante y el cual presenta un sinnúmero de enfermedades asociadas, como problemas cardiovasculares, diabetes, trastornos ósteomusculares y algunos cánceres; este último viene a ser el de mayor riesgo a salud por su complejidad. Según estadísticas de los EE.UU. para el rango de sobrepeso $\left(25-30 \mathrm{~d} » \mathrm{~kg}^{*} \mathrm{~m}^{-2}\right)$ la prevalencia ronda el 39\% para hombres y 27\% para mujeres; más aún, la obesidad (e»30 $\mathrm{kg}^{*} \mathrm{~m}^{-2}$ ) se presenta en $38 \%$ de los hombres y $40 \%$ de las mujeres (Jakicic, et al., 2019), evidenciando el alto porcentaje que posiblemente pueda haber en otras regiones del planeta como Latinoamérica, donde los hábitos saludables de realizar actividad física como prevención y regulación del peso corporal podrían ser muy limitados. Caso concreto se evidencia en el Estudio Latinoamericano de Nutrición y Salud (ELANS) publicado en el año 2016, el cual determinó que Costa Rica es el segundo país de América Latina con mayor prevalencia de sobrepeso y obesidad: el 32,6\% de la población entre 15-65 años presentan sobrepeso y el 30,6\% presentaron obesidad según el Índice de Masa Corporal (IMC), siendo 2 de cada 3 hombres y mujeres posee- 
dores de un exceso de peso (El Mundo CR, 2018) Asimismo, la encuesta nacional de nutrición de Costa Rica proporciona datos a tomar en cuenta: con relación a la prevalencia de obesidad en niños (as) de 5-12 años y en adultos costarricenses, se nota un incremento constante desde 1982 hasta la fecha de la encuesta (2009), pues se pasó del $14.9 \%$ al $21.4 \%$ en niños (as) y de $45.9 \%$ a $59.7 \%$ en mujeres de 20 44 años (Ávila , 2009).

Aunque la actividad física es de utilidad limitada para bajar de peso, es una herramienta muy importante para prevenir el aumento de peso. Según Gibbs et al. (2017), la ganancia de peso por año ronda los 0,5-0,8 kg siendo un aumento natural y constante cuando no se realiza suficiente actividad física (Gibbs, et al., 2017). La acumulación año a año de peso, trae consigo problemas de salud que en edades avanzadas son de mayor riesgo; no obstante, realizar actividad física a intensidad moderada a vigorosa en estas estas edades, presenta resultados positivos para la prevención y disminución de peso (Botoseneanu \& Liang, 2012; Brown, Kabir, Clark, \& Gomersall, 2016; Williams \& Wood, 2006). Esta afirmación está sustentada en los lineamientos de realizar por lo menos $150 \mathrm{~min}^{*}$ semana $^{-1}$ de actividad física de intensidad moderada o lograr 10.000 pasos por día, para intentar neutralizar el aumento de peso o para evitar aumentos en el IMC (Hamer, et al., 2013; Smith, et al., 2017). Sin embargo, otros estudios señalan que mayores cantidades de actividad física reducen con mayor efectividad los efectos del aumento de peso, con al menos 167 min*semana $^{-1}$ de actividad física de intensidad moderada, que equivale a e» 500 METmin*semana (Brown, et al., 2016), o más de 300 min* semana $^{-1}$ de actividad física de intensidad vigorosa que equivale a $>900$ MET-min*semana (Blanck, et al., 2007). Aun cuando no necesariamente modifica el peso corporal, se ha sugerido que el aumento de cada 2000 pasos diarios podría reducir el progreso hacia la diabetes tipo II en un 5,5\% (Kraus, et al. 2019), un efecto también positivo sobre la salud.

La intensidad de la actividad física es una variable por tomar en cuenta para poder presentar resultados favorables. Rosenberg et al. (2013), evidencian que realizar por lo menos $1 \mathrm{~h} *_{\text {semana }}^{-1}$ de actividad física a una intensidad moderada, logra reducir el riesgo de desarrollar obesidad tanto en mujeres de peso normal como en mujeres con sobrepeso. Asimismo, se considera tener una menor duración si la intensidad pasa de moderada a vigorosa (Rosenberg,
Kipping-Ruane, Boggs, \& Palmer, 2013). Williams y Wood (2006) toman en cuenta la ganancia de peso que se presenta en adultos y adultos mayores y señalan que, bajo la perspectiva de realizar actividad física de carrera a intensidad vigorosa, se debería tomar en cuenta un aumento de 2,7 y 3,9 km semanales cada año, para compensar la ganancia anual de peso por envejecimiento que pueden tener hombres y mujeres, respectivamente.

Moholdt et al. (2014) analizan la prevención de aumento de peso en $2 \mathrm{~kg}$ teniendo en comparativa con cuatro grupos: inactivo; por debajo de las recomendaciones ( $<150 \mathrm{~min} *$ semana de actividad física); según las recomendaciones, 150 min* semana; por arriba de las recomendaciones (> $150 \mathrm{~min} *$ semana). Usando como referencia al grupo inactivo, resaltan que el riesgo relativo de ganar $2 \mathrm{~kg}$ para la categoría «conforme a las recomendaciones» fue de 0.97 con intervalos de confianza al $95 \%(0,87-1,08)$ y para la categoría «sobre recomendaciones» 0.79 (IC 95\% 0,69-0,91), para los hombres. En similares condiciones observado en mujeres (0,97, IC 95\% 0,88 - 1,07; 0,69, IC 95\% 0,59 - 0,82) (Moholdt, Wisløff, Lydersen, \& Nauman, 2014). Estos hallazgos incentivan a recomendar el aumento en tiempo por encima de las recomendaciones establecidas de $150 \min _{*}$ semana $^{-1}$ de actividad física, para lograr un beneficio más certero en lo concerniente a la prevención de la ganancia de peso.

\section{Actividad física y función cognitiva}

El envejecimiento trae consigo una serie de cambios a nivel cognitivo. Lorenzo Otero y Fontán Scheitler (2003) afirman que, en ausencia de una enfermedad neurológica o sistémica, el adulto mayor sano presenta un deterioro pequeño en el área cognitiva, deteriorándose primero las habilidades visuales y espaciales, de cálculo y de aprendizaje, mientras que se conservan mejor las habilidades verbales y conocimientos generales, denominado déficit cognitivo leve, el cual va a presentar en algún momento de la vida del adulto mayor. A su vez, a partir de los 60 años se presenta un deterioro cognitivo que aumenta el doble cada 5 años comenzando en un 10\% hasta un $45 \%$ a una edad de 85 años. A manera de ejemplo, Costa Rica ha evidenciado por medio del informe del Consejo Nacional de la Persona Adulta Mayor del 2008, una prevalencia del $18 \%$ de adultos mayores con déficit cognitivo de los cuales hay una ligera elevación en mujeres que viven 
fuera del área Metropolitana (Sánchez-González \& Marín-Mora, 2016).

La función cognitiva es una variable de sumo interés dentro de las perspectivas estudiadas en relación con la actividad física, pues tiene el potencial de presentar profundos efectos en la actividad cerebral que influyen en una calidad de vida óptima. Un claro ejemplo de la influencia de la actividad física sobre la función cognitiva y que ha sido profundamente estudiada es la reducción del riesgo de deterioro cognitivo que se presenta a medida que avanza la edad, el cual se cataloga como deterioro cognitivo leve al no padecer de ninguna afectación; sin embargo, si hay incapacidad para realizar actividades de la vida diaria, se clasifica como demencia leve, moderada o grave. Al padecer de una afectación a nivel cognitivo, es importante entender el principio neurobiológico de los sucesos celulares y moleculares en el cerebro, el cual logra modificarse por el enriquecimiento ambiental (Kobilo, et al., 2011). Un ambiente favorable y motivacional, es realizar actividad física donde se logran cambios positivos en la función cognitiva (Kelly, et al., 2014).

Diversos estudios han analizado la influencia que puede tener la actividad física en la función cognitiva en seres humanos, donde se logran establecer rangos por edad y su efectividad (Erickson, et al., 2019). Dentro de los resultados observados, se destaca la población que presenta riesgo de demencia y deterioro (adultos de más de 50 años): en estas personas, una mayor cantidad de actividad física produce una disminución significativa el deterioro cognitivo. Asimismo, hay evidencia fuerte y significativa para afirmar que los episodios cortos y agudos de actividad física de intensidad moderada a vigorosa en adultos mayores, logran una mejoría transitoria en la cognición durante los periodos post recuperación (Erickson et al., 2019, p. 1244). Adicionalmente, existe evidencia moderada para afirmar la influencia de la actividad física moderada - vigorosa sobre la mejora de la cognición, esto incluyendo el rendimiento en pruebas de rendimiento académico (6-13 años de edad), así como pruebas neuropsicológicas aplicadas a la población en general (Erickson, et al., 2019).

A medida que las personas avanzan en su edad cronológica, se presentan cambios naturales tanto a nivel físico como cognitivo; estos cambios, logran ser llevados de una mejor manera, cuando la persona presenta hábitos saludables como lo es realizar actividad física; en el rango de edades en que más se investigan estos efectos $(18-65)$, el factor común en las conclusiones se centra en el efecto positivo de la actividad física sobre la función cognitiva y su protección ante posibles enfermedades. Northey et al. (2018) sugieren tener un programa de ejercicios con componentes de entrenamiento de tipo aeróbico y de resistencia, de intensidad moderada en promedio de 45 minutos por sesión, tantos días de la semana como sea posible, logrando beneficios en la función cognitiva que se hacen evidentes en los adultos a partir de los 50 años (Northey, Cherbuin, Pumpa, Smee, \& Rattray., 2018).

\section{Actividad física y osteoartritis}

Existen alrededor de cien condiciones de artritis, entre las cuales se encuentra la osteoartritis, uno de los trastornos articulares más comunes en la población. La osteoartritis llega a ocupar el 13,4\% en los adultos estadounidenses (Kraus, et al., 2019). La osteoartritis de rodilla viene a ser uno de los mayores padecimientos de la población estadounidense, en lo referente a problemas articulares, asimismo, se espera que para el año 2040, 78 millones (25,9\% de la población adulta total proyectada) tengan artritis diagnosticada por un médico, siendo en su mayoría relacionada con osteoartritis (Hootman, Helmick, Barbour, Theis, \& Boring, 2016).

Existe evidencia favorable para afirmar que la actividad física reduce los problemas ocasionados por la osteoartritis en las extremidades inferiores; al realizar actividades físicas de Tai chi, se producen reducciones significativas de dolor para rodilla y cadera (Chang, Chen, Lee, Lin, \& Lai, 2016). Estas reducciones se han mantenido a través del tiempo hasta llegar a los 6 meses con una reducción de dolor. También, se destaca el efecto del ejercicio aeróbico sobre el alivio del dolor con un mayor número de sesiones supervisadas; en general, se produce mayor reducción del dolor cuando se realizaba ejercicio supervisado al menos tres veces por semana; tomando en cuenta que realizar alrededor de 10.000 pasos por día en las diversas actividades, no acelera la osteoartritis progresiva en sujetos con osteoartritis preexistente, indistintamente del espacio donde se realice la actividad. Es importante señalar que realizar un total de al menos 45 min $^{*}$ semana $^{-1}$ de actividad física moderada a vigorosa puede mejorar o mantener la función de las extremidades inferiores en las personas con osteoartritis. 


\section{Conclusiones y recomendaciones finales}

Los beneficios para la salud que proporciona la actividad física, son importantes hasta el punto de cambiar la vida de las personas que hasta la fecha mantienen hábitos sedentarios. A su vez, para personas que ya presentan diversas enfermedades como diabetes, hipertensión, demencia o cáncer, realizar un mínimo de actividad física según el lineamiento establecido de 150 min de actividad por semana a intensidad moderada, proporciona beneficios; es recomendable tomar en cuenta las especificaciones de la actividad física a realizar y la intensidad donde se logren obtener los mejores resultados. Así, en la actividad física como en muchos aspectos de la vida diaria, la constancia y planificación son la ruta ideal para cumplir los objetivos propuestos.

Es importante resaltar que la actividad física regular genera una menor carga financiera para la atención de la salud en las personas, siendo este un punto importante para cualquier persona, sirviendo como una recomendación financiera y de salud. Por otra parte, la reducción de riesgo por muerte de enfermedades como diabetes e hipertensión, son significativas al seguir una rutina de intensidad moderada a vigorosa independientemente del tipo de actividad, siempre y cuando la actividad sea planificada o dirigida por un profesional que tenga el conocimiento del padecimiento que la persona presenta.

El estilo de vida de hoy, para un número importante de personas, implica pasar muchas horas sentadas o estar mucho tiempo frente a una pantalla. Este es un problema social que afecta la salud de las personas sin importar la edad, suscitando o promoviendo enfermedades como diabetes tipo 2 o enfermedad cardiovascular. El problema es especialmente crítico para las personas que permanecen en alguna de estas actividades durante 4 o más horas diarias, pues es claro que su riesgo de mortalidad prematura aumenta, especialmente cuanto menor es la actividad física diaria. En otras palabras: la actividad física regular puede compensar parcialmente los efectos nocivos del «tiempo silla»y el «tiempo pantalla», pero cuantas más horas diarias de sedentarismo experimente, tanto más prolongada deberá ser la actividad física de moderada a vigorosa. Es importante tomar en cuenta las recomendaciones de Ekelund et al. (2016, p. 1308) de realizar niveles elevados de actividad física (60-75 minutos por día) especialmente si los tiempos de pantalla no se reducen al mínimo po- sible $(<1$ hora diaria). También vale la pena mencionar el valor de un aporte de nuestra era tecnológica, la utilización de «wearables», dispositivos para un monitoreo constante y de recordación de objetivos diarios como lo es lograr 10.000 pasos por día que han demostrado su utilidad.

Lograr identificar las estrategias más efectivas para incentivar la actividad física en toda la población, es la recompensa de todo investigador que trabaje con la disminución del sedentarismo. La autosupervisión del comportamiento y la formación de la intención son algunas de las estrategias con mejores resultados para promover la iniciación de hábitos de actividad física, para cambiar el comportamiento sedentario. Los distintos grupos de edad como jóvenes y adultos mayores se ven beneficiados con intervenciones diferentes: para los jóvenes, la educación personal y las actividades experienciales son la mejor opción; en cambio, para adultos mayores, las intervenciones dirigidas a sus necesidades y gustos son las de mejores resultados, siempre y cuando se mantengan por un periodo mínimo de un año. Un punto a resaltar son las intervenciones en la etapa escolar para promover la actividad física y eventualmente disminuir el sedentarismo que en muchos casos trae la edad. En este contexto, las actividades multicomponente como «CATCH» $\mathrm{y}$ «SPARK», mencionadas en este documento, son las de mayor efectividad, siempre que sean dirigidas por profesionales, pues mejoran los tiempos de aprendizaje de los participantes y los llevan de niveles moderados a vigorosos de actividad física.

Los niveles de evidencia para respaldar los beneficios de la actividad física regular varían ampliamente según la enfermedad bajo estudio: la reducción en la mortalidad prematura o en la incidencia de enfermedades cardiovasculares está sólidamente demostrada, pero los beneficios sobre la osteoartritis o el deterioro cognitivo son menos claros. Existen pautas claramente establecidas de actividad física regular que brindan beneficios bien documentados. Realizar un mínimo de 150 minutos de actividad física por semana sigue siendo la recomendación mínima con la cual se logran cambios positivos en la salud. Sin embargo, la intensidad de esa actividad física es una variable de discusión en muchas investigaciones que toman este valor de referencia. La intensidad moderada a vigorosa es la de mayor aceptación y donde son más claros los beneficios para las distintas complicaciones que trae consigo el sedentarismo, en par- 
ticular las enfermedades cardiovasculares y la mortalidad prematura. Frente a problemas cardiovasculares y durante el embarazo, realizar 150 min de actividad física por semana disminuye significativamente los riesgos asociados. A su vez, al aumentar al doble la actividad física semanal (300 min*semana) se obtienen mayores beneficios para disminuir el riesgo presente en enfermedades cardiovasculares hasta en un 20\%, también, realizar esta cantidad de minutos por semana reduce el riesgo de presentar diabetes tipo II. La hipertensión, padecimiento de alta prevalencia en la población costarricense, se ve controlada o reducida en sus riesgos hasta en un 33\%, al realizar actividades que produzcan $60 \mathrm{MET}^{*} \mathrm{~h}^{*}$ semana $^{-1}$ sin importar la intensidad o el tipo de actividad. Es claro que también se requieren estos niveles superiores de actividad física para controlar el peso corporal: la recomendación mencionada anteriormente se refiere a aumentarla por encima de $150 \min _{*}$ semana $^{-1}$. Es importante resaltar las intervenciones desarrolladas con HIIT para atacar el sobrepeso y otras complicaciones de forma eficiente y en períodos cortos. Este tipo de entrenamiento, con las precauciones previamente mencionadas, ofrece una alternativa atractiva, aunque hasta el momento lo que brinda la investigación es muy abierto, dejando lagunas que deben rellenarse con investigación más detallada.

Son muchas las intervenciones que se han puesto a prueba para intentar documentar una mejora en distintas enfermedades o su prevención. Algunas aplicaciones de la actividad física tienen validez o justificación aparente (más bien, se podría decir evidente), como es el caso de la actividad física regular en adultos mayores, la cual les permite mantener su independencia y funcionalidad por más tiempo. Sin embargo, la poca claridad o limitada evidencia para una correcta generalización de los resultados beneficiosos es una situación con la que se enfrenta la comunidad. A nivel de la función cognitiva, por ejemplo, la actividad física presenta evidencia moderada sobre sus beneficios, con poca claridad sobre las mejoras en pruebas de rendimiento académico en niños, así como ausencia de resultados significativos de un beneficio de la actividad física sobre pruebas neuropsicológicas en la población en general. Algunas enfermedades como la osteoartritis no presentan información relacionada con beneficios de la actividad física en miembros superiores del cuerpo, aunque sí se han documentado beneficios significati- vos de la práctica regular del Tai-Chi en las extremidades inferiores. Para algunas enfermedades como el Parkinson, aun no son claras las intervenciones necesarias para producir un beneficio significativo, a pesar de recomendarse el ejercicio multicomponente como una alternativa favorable. Finalmente, algunos tipos de cáncer mejoran considerablemente como resultado de la actividad física regular, pero la evidencia aún es muy limitada en los casos de cáncer de ovarios, páncreas, próstata, tiroides y recto, donde el porcentaje de reducción de mortalidad y la relación dosis-respuesta de la actividad física no ofrecen números sólidos para concluir posibles beneficios.

A toda esta información aquí recopilada y analizada, hay que añadir observaciones sobre algunas de las falencias observadas tanto en las diferentes publicaciones aquí citadas como el modo en que la información científica es transmitida e interiorizada por la población. Los beneficios de la actividad física frente a la salud son bien conocidos por la población en general; sin embargo, el lograr pasar de la generalidad a la individualidad proporcionaría un paso muy importante en la divulgación de formas y métodos de realizar la actividad física en el ámbito de salud. Asimismo, a nivel latinoamericano la falta de políticas públicas que promuevan la actividad física logrando la aceptación por parte de la población de forma asertiva y es un problema que aún se vive y debe ser desarrollado bajo parámetros tanto científicos como políticos para un desarrollo integral de los planes a desarrollar que promuevan la actividad física como medio de prevención.

\section{Agradecimientos}

Este manuscrito se preparó con financiamiento del proyecto VI-838-B6-766 «Observatorio del Sedentarismo» de la Universidad de Costa Rica.

\section{Referencias}

Ávila Agüero, M. L., (2009). Encuesta Nacional de Nutrición, Costa Rica, 2008-2009. Ministerio de Salud, Instituto Costarricense de Investigación y Enseñanza en Nutrición y Salud, Caja Costarricense de Seguro Social, Instituto Nacional de Estadística y Censos, Instituto Costarricense sobre Drogas, INCAP/OPS. Recuperado de https://www.paho.org/cor/

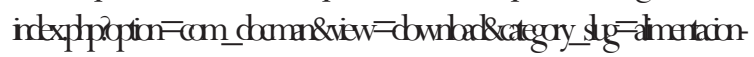
$\mathrm{y}$-nutricion \&alias $=67$ - encuesta - nacional - de- 
nutricion-costa-rica-2008-2009\&Itemid=222

Alwan, A., Armstrong, T., Bettcher, D., Branca, F., Chisholm, D., Ezzati, M., \& Wild, C. (2011). Informe sobre la situación mundial de las enfermedades no transmisibles 2010: Resumen de orientación. Ginebra: Organización Mundial de la Salud. Recuperado de https://www.who.int/nmh/ publications/ncd_report_summary_es.pdf

American College of Obstetricians and Gynecologists. (2017). Physical activity and exercise during pregnancy and the postpartum period. 2015. Committee opinion, 650. doi: 10.1097/ AOG.0000000000001214

Artal, R. (2003). Exercise during pregnancy and the postpartum period. Clin Obstet Gynecol, 46(2), 496499. doi: 10.1097/00003081-200306000-00028

Batacan, R. B., Duncan, M. J., Dalbo, V. J., Tucker, P. S., \& Fenning, A. S. (2017). Effects of highintensity interval training on cardiometabolic health: A systematic review and meta-analysis of intervention studies. Br J Sports Med, 51(6), 494503. doi: 10.1136/bjsports-2015-095841

Benjamin, E., Blaha, M., Chiuve, S., Cushman, M., Das, S., Deo, R., \& Muntner, P. (2017). Resumen de estadísticas de 2017 Enfermedad del corazón y ataque cerebral. Revista Circulation, 135(10), e146-e603. Recuperado de https:// professional.heart.org/idc/groups/ahamahpublic/@wcm/@sop/@smd/documents / downloadable/ucm_491392.pdf

Behrens, G., Jochem, C., Keimling, M., Ricci, C., Schmid, D., \& Leitzmann, M. F. (2014). The association between physical activity and gastroesophageal cancer: Systematic review and meta-analysis. European journal of epidemiology, 29(3), 151-170. doi: 10.1007/s10654-014-98952

Bizzozero, P., \& Díaz, G. (2019). Efectos del entrenamiento por intervalos vs entrenamiento continuo sobre capacidad aeróbica en pacientes con enfermedad de las arterias coronarias. Pensar en Movimiento: Revista de Ciencias del Ejercicio y la Salud, 17(2), e37766-e37766. doi: 10.15517/ PENSARMOV.V17I2.37766

Blanck, H. M., McCullough, M. L., Patel, A. V., Gillespie, C., Calle, E. E., Cokkinides, V. E., Galuska, D. A., Khan, L. K., \& Serdula, M. K. (2007). Sedentary behavior, recreational physical activity, and 7 year weight gain among postmenopausal US women. Obesity, 15(6), 1578-
1588. doi: $10.1038 /$ oby. 2007.187

Bonn, S. E., Sjölander, A., Lagerros, Y. T., Wiklund, F., Stattin, P., Holmberg, E., Grönberg, H., \& Bälter, K. (2015). Physical activity and survival among men diagnosed with prostate cancer. Cancer Epidemiology and Prevention Biomarkers, 24(1), 5764. doi: 10.1158/1055-9965.EPI-14-0707

Botoseneanu, A., \& Liang, J. (2012). The effect of stability and change in health behaviors on trajectories of body mass index in older Americans: A 14-year longitudinal study. Journals of Gerontology Series A: Biomedical Sciences and Medical Sciences, 67(10), 1075-1084. doi: 10.1093/gerona/ gls073

Brown, W. J., Kabir, E., Clark, B. K., \& Gomersall, S. R. (2016). Maintaining a healthy BMI: data from a 16-year study of young Australian women. American journal of preventive medicine, 51(6), e165e178. doi: 10.1016/j.amepre.2016.09.007

Campbell,W.W., Kraus, W. E., Powell, K. E., Haskell, W. L., Janz, K. F., Jakicic, J. M., Troiano, R. P., Sprow, K., Torres, A., \& Piercy, K. L. (2019). High-intensity interval training for cardiometabolic disease prevention. Medicine \& Science in Sports \& Exercise, 51(6), 1220-1226. doi: 10.1249/MSS.0000000000001934

Cauley, J. A., Harrison, S. L., Cawthon, P. M., Ensrud, K. E., Danielson, M. E., Orwoll, E., \& Mackey, D. C. (2013). Objective measures of physical activity, fractures and falls: The osteoporotic fractures in men study. Journal of the American Geriatrics Society, 61(7), 1080-1088. doi: 10.1111/jgs.12326 Chang, W.-D., Chen, S., Lee, C.-L., Lin, H.-Y., \& Lai, P.-T. (2016). The effects of tai chi chuan on improving mind-body health for knee osteoarthritis patients: A systematic review and meta-analysis. Evidence-Based Complementary and Alternative Medicine, 2016. doi: 10.1155/2016/ 1813979

Chau, J.Y., Grunseit, A. C., Chey, T., Stamatakis, E., Brown, W. J., Matthews, C. E., Bauman, A. E., \& van der Ploeg, H. P. (2013). Daily sitting time and all-cause mortality: A meta-analysis. PloS one, 8(11), e80000. doi: $10.1371 /$ journal.pone.0080000

Chobanian, A. (2003). National heart, lung, and blood institute; national high blood pressure education program coordinating committee. Seventh report of the joint national committee on prevention, detection, evaluation, and treatment of high blood 
pressure. Hypertension, 42, 1206-1252. doi: 10.1161/01.HYP.0000107251.49515.c2

Cigarroa, I., Sarqui, C., \& Lamana, R. Z. (2016). Efectos del sedentarismo y obesidad en el desarrollo psicomotor en niños y niñas: Una revisión de la actualidad latinoamericana. Universidad y Salud, 18(1), 156-169. doi: 10.22267/ rus. 161801.27

Cogliano, V. J., Baan, R., Straif, K., Grosse, Y., LaubySecretan, B., El Ghissassi, F., Bouvard, V., Benbrahim-Tallaa, L., Guha, N., \& Freeman, C. (2011). Preventable exposures associated with human cancers. Journal of the National Cancer Institute, 103(24), 1827-1839. doi: 10.1093/jnci/ djr 483

Crespo-Salgado, J. J., Delgado-Martín, J. L., BlancoIglesias, O., \& Aldecoa-Landesa, S. (2015). Guía básica de detección del sedentarismo y recomendaciones de actividad física en atención primaria. Atención primaria, 47(3), 175-183. doi: 10.1016/ j.aprim.2014.09.004

Cushing, C. C., Brannon, E. E., Suorsa, K. I., \& Wilson, D. K. (2014). Systematic review and meta-analysis of health promotion interventions for children and adolescents using an ecological framework. Journal of Pediatric Psychology, 39(8), 949-962. doi: 10.1093/jpepsy/jsu042

da Silva, S. G., Ricardo, L. I., Evenson, K. R., \& Hallal, P. C. (2017). Leisure-time physical activity in pregnancy and maternal-child health: A systematic review and meta-analysis of randomized controlled trials and cohort studies. Sports Medicine, 47(2), 295-317. doi: 10.1007/ s40279-016-0565-2

del Castillo, M. (2011). La actividad física durante el embarazo. En In Estudios multidisciplinares para la humanización del parto (pp. 137-146). Servizo de Publicacións. Recuperado de https: / / ruc.udc.es/ dspace/bitstream/handle/2183/9092 / CC1 19. art8.pdf?sequence $=1$ \&isAllowed $=\mathrm{y}$

Deputy, N. P., Sharma, A. J., \& Kim, S. Y. (2015). Gestational weight gain-United States, 2012 and 2013. MMWR. Morbidity and mortality weekly report, 64(43), 1215. doi: 10.15585/mmwr.mm6443a3

DeSisto, C. L., Kim, S. Y., \& Sharma, A. J. (2014). Peer reviewed: Prevalence estimates of gestational diabetes mellitus in the United States, pregnancy risk assessment monitoring system (prams), 2007-2010. Preventing chronic disease, 11. doi: $10.5888 / \operatorname{pcd} 11.130415$ de Rezende, L. F. M., de Sá, T. H., Markozannes, G., Rey-López, J. P., Lee, I.-M., Tsilidis, K. K., Ioannidis, J. P., \& Eluf-Neto, J. (2018). Physical activity and cancer: An umbrella review of the literature including 22 major anatomical sites and 770000 cancer cases. British journal of sports medicine, 52(13), 826-833. doi: 10.1136/bjsports2017-098391

Díaz, I. A., Reche, M. P. C., Torres, J. M. T., \& Rodríguez, J. M. R. (2019). Impacto de las apps móviles en la actividad física: Un meta-análisis (Impact of mobile apps on

physical activity: A meta-analysis). Retos: nuevas tendencias en educación física, deporte y recreación, 36, 52-57. doi: https://doi.org/ $10.47197 /$ retos.v36i36.66628

Ding, D., Lawson, K. D., Kolbe-Alexander, T. L., Finkelstein, E. A., Katzmarzyk, P. T., Van Mechelen, W., Pratt, M., \& Lancet Physical Activity Series 2 Executive Committee. (2016a). The economic burden of physical inactivity: A global analysis of major non-communicable diseases. The Lancet, 388(10051), 1311-1324. doi: 10.1016/S0140-6736(16)30383-X

Dipietro, L., Campbell, W. W., Buchner, D. M., Erickson, K. I., Powell, K. E., Bloodgood, B., Hughes, T., Day, K. R., Piercy, K. L., \& VauxBjerke, A. (2019). Physical activity, injurious falls, and physical function in aging: An umbrella review. Medicine \& Science in Sports \& Exercise, 51(6), 13031313. doi: 10.1249/MSS.0000000000001942

Ekelund, U., Steene-Johannessen, J., Brown, W. J., Fagerland, M. W., Owen, N., Powell, K. E., Bauman, A., \& Lee, I.-M. (2016). Does physical activity attenuate, or even eliminate, the detrimental association of sitting time with mortality? A harmonised meta-analysis of data from more than 1 million men and women. The Lancet, 388(10051), 1302-1310. doi: 10.1016/ S0140-6736(16)30370-1

El Mundo CR. (2018, noviembre 12). Costa Rica es el segundo país con mayor obesidad de América Latina. El Mundo CR. Recuperado de https:// www.elmundo.cr/costa-rica/costa-rica-es-el-segundo-pais-con-mayor-obesidad-de-america-latina/

El-Khoury, F., Cassou, B., Charles, M.-A., \& Dargent-Molina, P. (2013). The effect of fall prevention exercise programmes on fall induced injuries in community dwelling older adults: 
Systematic review and meta-analysis of randomised controlled trials. $B M j$, 347, f6234. doi: 10.1136/bmj.f6234

Erickson, K. I., Hillman, C., Stillman, C. M., Ballard, R. M., Bloodgood, B., Conroy, D. E., Macko, R., Marquez, D. X., Petruzzello, S. J., \& Powell, K. E. (2019). Physical activity, cognition, and brain outcomes: A review of the 2018 physical activity guidelines. Medicine \& Science in Sports \& Exercise, 51(6), 1242-1251. doi: 10.1249/ MSS.0000000000001936

Espinoza-Salinas, A., González-Jurado, J., BurdilesÁlvarez, A., Arenas-Sánchez, G., \&

Bobadilla-Olivares, M. (2020). Efectos del entrenamiento cardiovagal en la respuesta autonómica en personas con sobrepeso Effects of cardiovagal training on autonomic response in overweight people. Retos, 38, 118-122. doi: https://doi.org/10.47197/retos.v38i38.73994

Evenson, K. R., Mottola, M. F., Owe, K. M., Rousham, E. K., \& Brown, W. J. (2014). Summary of international guidelines for physical activity following pregnancy. Obstetrical \& gynecological survey, 69(7), 407. doi: 10.1097/ OGX.0000000000000077

Facts About Healthy Aging. (2015, junio 3). NCOA. Recuperado de https://www.ncoa.org/news/ resources-for-reporters/get-the-facts/healthyaging-facts/

FMI. (2013). Report for Selected Countries and Subjects. Recuperado de https://www.imf.org/external/ pubs/ft/weo/2015/02/weodata/

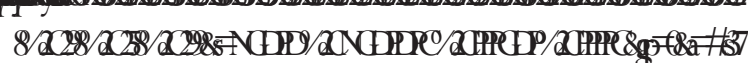

French, D. P., Olander, E. K., Chisholm, A., \& Mc Sharry, J. (2014). Which behaviour change techniques are most effective at increasing older adults' self-efficacy and physical activity behaviour? A systematic review. Annals of behavioral medicine, 48(2), 225-234. doi: 10.1007/ s12160-014-9593-z

Gibbs, B. B., Gabriel, K. P., Carnethon, M. R., GaryWebb, T., Jakicic, J. M., Rana, J. S., Reis, J. P., Siddique, J., Sternfeld, B., \& Lewis, C. E. (2017). Sedentary time, physical activity, and adiposity: Cross-sectional and longitudinal associations in CARDIA. American journal of preventive medicine, 53(6), 764-771. doi: 10.1016/ j.amepre.2017.07.009

Gijón-Conde, T., Gorostidi, M., Camafort, M., Abad-
Cardiel, M., Martín-Rioboo, E., Morales-Olivas, F., ... \& De La Sierra, A. (2018). Documento de la Sociedad Española de Hipertensión-Liga Española para la Lucha contra la Hipertensión Arterial (SEH- LELHA) sobre las guías ACC/AHA 2017 de hipertensión arterial. Hipertensión y riesgo vascular, 35(3), 119-129.

Guthold, R., Stevens, G. A., Riley, L. M., \& Bull, F. C. (2018). Worldwide trends in insufficient physical activity from 2001 to 2016: A pooled analysis of 358 population-based surveys with 1 . 9 million participants. The lancet global health, 6(10), e1077-e1086. doi: 10.1016/S2214109X(18)30357-7

Hallal, P. C., Andersen, L. B., Bull, F. C., Guthold, R., Haskell, W., Ekelund, U., \& Lancet Physical Activity Series Working Group. (2012). Global physical activity levels: Surveillance progress, pitfalls, and prospects. The lancet, 380(9838), 247257. doi: 10.1016/S0140-6736(12)60646-1

Hamer, M., Brunner, E., Bell, J., Batty, G. D., Shipley, M., Akbaraly, T., Singh Manoux, A., \& Kivimaki, M. (2013). Physical activity patterns over 10 years in relation to body mass index and waist circumference: The Whitehall II cohort study. Obesity, 21(12), E755-E761. doi: 10.1002/ oby. 20446

Hamer, M., \& Chida,Y. (2008). Walking and primary prevention: A meta-analysis of prospective cohort studies. British journal of sports medicine, 42(4), 238 243. doi: 10.1136/bjsm.2007.039974

Hesketh, K. R., \& Evenson, K. R. (2016). Prevalence of US pregnant women meeting 2015 ACOG physical activity guidelines. American journal of preventive medicine, 51(3), e87-e89. doi: 10.1016/ j.amepre. 2016.05.023

Hootman, J. M., Helmick, C. G., Barbour, K. E., Theis, K. A., \& Boring, M. A. (2016). Updated projected prevalence of self reported doctor diagnosed arthritis and arthritis attributable activity limitation among US adults, 2015-2040. Arthritis \& rheumatology, 68(7), 1582-1587. doi: 10.1002/art.39692

Hospital Clínica Bíblica. (2015). 34 \% costarricenses padece de hipertensión arterial. Hospital Clínica Bíblica. Recuperado de https:// www.clinicabiblica.com/es/component/ content/article?id=2039:34-costarricenses-padece-de-hipertension-arterial

International Agency for Research on Cancer. (2018). 
All cancers source: Globocan 2018 (pp. 1-2). Recuperado de https://gco.iarc.fr/today/data/ factsheets / cancers /39-All-cancers-fact-sheet.pdf Jakicic, J. M., Powell, K. E., Campbell, W. W., Dipietro, L., Pate, R. R., Pescatello, L. S., Collins, K. A., Bloodgood, B., \& Piercy, K. L. (2019). Physical Activity and the Prevention of Weight Gain in Adults: A Systematic Review. Medicine and science in sports and exercise, 51(6), 1262-1269. doi: 10.1249/MSS.0000000000001938

Jelleyman, C., Yates, T., O’Donovan, G., Gray, L. J., King, J. A., Khunti, K., \& Davies, M. J. (2015). The effects of high intensity interval training on glucose regulation and insulin resistance: A meta analysis. Obesity reviews, 16(11), 942-961. doi: 10.1111 /obr. 12317

Keimling, M., Behrens, G., Schmid, D., Jochem, C., \& Leitzmann, M. (2014). The association between physical activity and bladder cancer: Systematic review and meta-analysis. British journal of cancer, 110(7), 1862-1870. doi: 10.1038/bjc. 2014.77

Kelly, M. E., Loughrey, D., Lawlor, B. A., Robertson, I. H., Walsh, C., \& Brennan, S. (2014). The impact of exercise on the cognitive functioning of healthy older adults: a systematic review and metaanalysis. Ageing research reviews, 16, 12-31. doi: 10.1016/j.arr.2014.05.002

Kelly, P., Kahlmeier, S., Götschi, T., Orsini, N., Richards, J., Roberts, N., Scarborough, P., \& Foster, C. (2014). Systematic review and metaanalysis of reduction in all-cause mortality from walking and cycling and shape of dose response relationship. International journal of behavioral nutrition and physical activity, 11(1), 132. doi: 10.1186/s12966-014-0132-x

Kessler, H. S., Sisson, S. B., \& Short, K. R. (2012). The potential for high-intensity interval training to reduce cardiometabolic disease risk. Sports medicine, 42(6), 489-509. doi: 10.2165/11630910000000000-00000

Kim, Y., Wilkens, L. R., Park, S.-Y., Goodman, M. T., Monroe, K. R., \& Kolonel, L. N. (2013). Association between various sedentary behaviours and all-cause, cardiovascular disease and cancer mortality: The Multiethnic Cohort Study. International Journal of Epidemiology, 42(4), 1040 1056. doi: 10.1093/ije/dyt108

King, A. C., Whitt-Glover, M. C., Marquez, D. X., Buman, M. P., Napolitano, M. A., Jakicic, J., Fulton, J. E., \& Tennant, B. L. (2019). Physical
Activity Promotion: Highlights from the 2018 Physical Activity Guidelines Advisory Committee Systematic Review. Medicine and science in sports and exercise, 51(6), 1340-1353. doi: 10.1249/ mss.0000000000001945

Kinnafick, F.-E., Thøgersen-Ntoumani, C., Shepherd, S. O., Wilson, O. J., Wagenmakers, A. J., \& Shaw, C. S. (2018). In it together: A qualitative evaluation of participant experiences of a 10 week, group-based, workplace HIIT program for insufficiently active adults. Journal of Sport and Exercise Psychology, 40(1), 10-19. doi: 10.1123/ jsep.2017-0306

Kobilo, T., Liu, Q.-R., Gandhi, K., Mughal, M., Shaham, Y., \& van Praag, H. (2011). Running is the neurogenic and neurotrophic stimulus in environmental enrichment. Learning \& memory, 18(9), 605-609. doi: 10.1101/lm.2283011

Kraus V.B., Sprow, K., Powell, K. E., Buchner, D., Bloodgood, B., Piercy, K., George, S. M., \& Kraus, W. E. (2019). Effects of Physical Activity in Knee and Hip Osteoarthritis: A Systematic Umbrella Review. Medicine and science in sports and exercise, 51(6), 1324-1339. doi: 10.1249/ MSS.0000000000001944

Kraus, W. E., Powell, K. E., Haskell, W. L., Janz, K. F., Campbell, W. W., Jakicic, J. M., Troiano, R. P., Sprow, K., Torres, A., \& Piercy, K. L. (2019). Physical Activity, All-Cause and Cardiovascular Mortality, and Cardiovascular Disease. Medicine and science in sports and exercise, 51(6), 1270-1281. doi: 10.1249/MSS.0000000000001939

Law, M., Morris, J., \& Wald, N. (2009). Use of blood pressure lowering drugs in the prevention of cardiovascular disease: Meta-analysis of 147 randomised trials in the context of expectations from prospective epidemiological studies. Bmj, 338, b1665. doi: 10.1136/bmj.b1665

Lee, I.-M., Shiroma, E. J., Lobelo, F., Puska, P., Blair, S. N., Katzmarzyk, P. T., \& Lancet Physical Activity Series Working Group. (2012). Effect of physical inactivity on major non-communicable diseases worldwide: An analysis of burden of disease and life expectancy. The lancet, 380(9838), 219-229. doi: 10.1016/S0140-6736(12)61031-9

Liu, L., Shi, Y., Li, T., Qin, Q., Yin, J., Pang, S., Nie, S., \& Wei, S. (2016). Leisure time physical activity and cancer risk: Evaluation of the WHO's recommendation based on 126 high-quality epidemiological studies. British journal of sports 
medicine, 50(6), 372-378. doi: 10.1136/bjsports2015-094728

Liu, X., Zhang, D., Liu, Y., Sun, X., Han, C., Wang, B., Ren, Y., Zhou, J., Zhao, Y., \& Shi, Y. (2017). Dose-response association between physical activity and incident hypertension: A systematic review and meta-analysis of cohort studies. Hypertension, 69(5), 813-820. doi: 10.1161/ HYPERTENSIONAHA.116.08994

Lonsdale, C., Rosenkranz, R. R., Peralta, L. R., Bennie, A., Fahey, P., \& Lubans, D. R. (2013). A systematic review and meta-analysis of interventions designed to increase moderate-tovigorous physical activity in school physical education lessons. Preventive medicine, 56(2), 152 161. doi: 10.1016/j.ypmed.2012.12.004

López-Köstner, francisco, \& Zarate C, alejandro. (2012). El deporte y la actividad física en la prevención del cáncer. Revista Médica Clínica Las Condes, 23(3), 262-265. doi: 10.1016/S07168640(12)70309-7

Lorenzo Otero, J., \& Fontán Scheitler, L. (2003). Las fronteras entre el envejecimiento cognitivo normal y la enfermedad de Alzheimer.: El concepto de deterioro cognitivo leve. Revista médica del Uruguay, 19(1), 4-13. Recuperado de http:/ / www.scielo.edu.uy/pdf/rmu/v19n1/ v19n1a02.pdf

Luepker, R. V., Perry, C. L., McKinlay, S. M., Nader, P. R., Parcel, G. S., Stone, E. J., Webber, L. S., Elder, J. P., Feldman, H. A., \& Johnson, C. C. (1996). Outcomes of a field trial to improve children's dietary patterns and physical activity: The Child and Adolescent Trial for Cardiovascular Health (CATCH). Jama, 275(10), 768-776. doi: 10.1001/jama.1996.03530340032026

Maillard, F., Pereira, B., \& Boisseau, N. (2018). Effect of high-intensity interval training on total, abdominal and visceral fat mass: A meta-analysis. Sports Medicine, 48(2), 269-288. doi: 10.1007/s40279017-0807-y

McTiernan, A., Friedenreich, C. M., Katzmarzyk, P. T., Powell, K. E., Macko, R., Buchner, D., Pescatello, L. S., Bloodgood, B., Tennant, B., \& Vaux-Bjerke, A. (2019). Physical Activity in Cancer Prevention and Survival: A Systematic Review. Medicine and science in sports and exercise, 51(6), 1252-1261. doi: 10.1249/ MSS.0000000000001937

Miller, K. D., Siegel, R. L., Lin, C. C., Mariotto, A.
B., Kramer, J. L., Rowland, J. H., Stein, K. D., Alteri, R., \& Jemal, A. (2016). Cancer treatment and survivorship statistics, 2016. CA: a cancer journal for clinicians, 66(4), 271-289. doi: 10.3322/ caac. 21349

Mitchell, M. S., Goodman, J. M., Alter, D. A., John, L. K., Oh, P. I., Pakosh, M. T., \& Faulkner, G. E. (2013). Financial incentives for exercise adherence in adults: Systematic review and metaanalysis. American journal of preventive medicine, 45(5), 658-667. doi: 10.1016/ j.amepre. 2013.06 .017

Moholdt, T., Wisløff, U., Lydersen, S., \& Nauman, J. (2014). Current physical activity guidelines for health are insufficient to mitigate long-term weight gain: More data in the fitness versus fatness debate (The HUNT study, Norway). Br J Sports Med, 48(20), 1489-1496. doi: 10.1136/ bjsports-2014-093416

Northey, J. M., Cherbuin, N., Pumpa, K. L., Smee, D. J., \& Rattray, B. (2018). Exercise interventions for cognitive function in adults older than 50: A systematic review with meta-analysis. Br J Sports Med, 52(3), 154-160. doi: 10.1136/bjsports-2016096587

Organización Panamericana de la Salud (OPS)/Organización Mundial de la Salud (OMS). (2014). Sistema de información regional de mortalidad 2014 (ICD-10: Enfermedad cardiovascular (I00-199), enfermedad cerebrovascular (I60-I69). Recuperado de https: / / www.paho.org/hq/dmdocuments / 2014/COSTA-RICA-PERFIL-ECV-2014.pdf

Pandey, A., Garg, S., Khunger, M., Darden, D., Ayers, C., Kumbhani, D. J., Mayo, H. G., de Lemos, J.A., \& Berry, J. D. (2015). Dose-response relationship between physical activity and risk of heart failure: A meta-analysis. Circulation, 132(19), 1786-1794. doi: 10.1161/ CIRCULATIONAHA.115.015853

Pescatello, L. S., Buchner, D. M., Jakicic, J. M., Powell, K. E., Kraus, W. E., Bloodgood, B., Campbell, W. W., Dietz, S., DiPietro, L., \& George, S. M. (2019). Physical activity to prevent and treat hypertension: A systematic review. Medicine \& Science in Sports \& Exercise, 51(6), 13141323. doi: 10.1249/MSS.0000000000001943

Physical Activity | Healthy People 2020. (s. f.). Recuperado de https://www.healthypeople.gov/ 2020/topics-objectives/topic/physical-activity Physical Activity Guidelines Advisory Committee. 
(2018). Physical activity guidelines advisory committee scientific report. Washington, DC: US Department of Health and Human Services, 2018, F233. Recuperado de https://health.gov/sites/ d e f a u l t / fil e s / 2019 - 9 / PAG_Advisory_Committee_Report.pdf

Rich-Edwards, J. W., Fraser, A., Lawlor, D. A., \& Catov, J. M. (2013). Pregnancy characteristics and women's future cardiovascular health: An underused opportunity to improve women's health? Epidemiologic reviews, 36(1), 57-70. doi: 10.1093/epirev/mxt006

Rosenberg, L., Kipping-Ruane, K. L., Boggs, D. A., \& Palmer, J. R. (2013). Physical activity and the incidence of obesity in young African-American women. American journal of preventive medicine, 45(3), 262-268. doi: 10.1016/ j.amepre.2013.04.016

Sabean Dixon, R., Aragón Vargas, L. F., \& Anderson Umaña, L. (2014). El juego. Una perspectiva cristiana. Cuaderno 1, ¿Por qué no jugamos? Las limitaciones del juego. http:// www.kerwa.ucr.ac.cr/handle/10669/596

Sallis, J. F., McKenzie, T. L., Alcaraz, J. E., Kolody, B., Faucette, N., \& Hovell, M. F. (1997). The effects of a 2-year physical education program (SPARK) on physical activity and fitness in elementary school students. Sports, Play and Active Recreation for Kids. American journal of public health, 87(8), 1328-1334. doi: 10.2105/ ajph.87.8.1328

Sánchez-González, L. R., \& Marín-Mora, A. (2016). Deterioro Cognitivo en adultos mayores Costa Rica: situación y factores de riesgo en centros diurnos. Revista Hispanoamericana de Ciencias de la Salud, 2(4), 288-293. Recuperado de http://www.uhsalud.com/index.php/ revhispano/article/view/213

Sattelmair, J., Pertman, J., Ding, E. L., Kohl III, H. W., Haskell, W., \& Lee, I.-M. (2011). Dose response between physical activity and risk of coronary heart disease: A meta-analysis. Circulation, 124(7), 789-795. doi: 10.1161/ CIRCULATIONAHA.110.010710

Schmid, D., Behrens, G., Keimling, M., Jochem, C., Ricci, C., \& Leitzmann, M. (2015). A systematic review and meta-analysis of physical activity and endometrial cancer risk. European journal of epidemiology, 30(5), 397-412. doi: 10.1007/ s10654-015-0017-6
Secretariat, M. A. (2008). Prevention of Falls and Fall-Related Injuries in Community-Dwelling Seniors: An Evidence-Based Analysis. Ontario Health Technology Assessment Series, 8(2), 1. Recuperado de https: / /www.ncbi.nlm.nih.gov/pmc/ articles/PMC3377567/pdf/ohtas-08-78.pdf

Sloth, M., Sloth, D., Overgaard, K., \& Dalgas, U. (2013). Effects of sprint interval training on VO $2 \max$ and aerobic exercise performance: A systematic review and meta analysis. Scandinavian journal of medicine \& science in sports, 23(6), e341e352. doi: 10.1111/sms. 12092

Smith, K. J., Gall, S. L., McNaughton, S. A., Cleland, V. J., Otahal, P., Dwyer, T., \& Venn, A. J. (2017). Lifestyle behaviours associated with 5 -year weight gain in a prospective cohort of Australian adults aged 26-36 years at baseline. BMC public health, 17(1), 54. doi: 10.1186/s12889-016-3931-y

Tremblay, M. S., Aubert, S., Barnes, J. D., Saunders, T. J., Carson, V., Latimer-Cheung, A. E., ... \& Chinapaw, M. J. (2017). Sedentary behavior research network (SBRN)- t e r m i n o l o g y consensus project process and outcome. International Journal of Behavioral Nutrition and Physical Activity, 14(1), 75. doi: 10.1186/s12966-017-0525-8Williams, P. T., \& Wood, P. D. (2006). The effects of changing exercise levels on weight and age-related weight gain. International journal of obesity, 30(3), 543. doi: $10.1038 /$ sj.ijo.0803172

van de Kop, J. H., van Kernebeek, W. G., Otten, R. H., Toussaint, H. M., \& Verhoeff, A. P. (2019). School-based physical activity interventions in prevocational adolescents: A systematic review and meta-analyses. Journal of Adolescent Health, 65(2), 185-194.

World Health Organization. (2020a). Physical activity. Recuperado de https://www.who.int/newsroom/fact-sheets/detail/physical-activity

World Health Organization. (2020b). WHO guidelines on physical activity and sedentary behaviour:At a glance. Recuperado de https://www.who.int/ publications/i/item/9789240015128

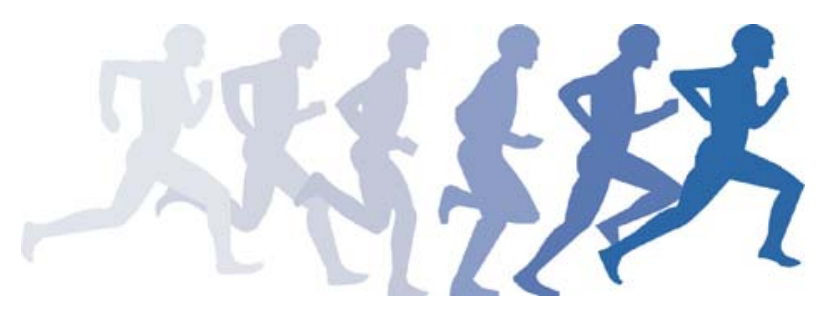

